\title{
Current Status and Future Perspectives of the LUCIFER Experiment
}

\author{
J. W. Beeman, ${ }^{1}$ F. Bellini, ${ }^{2,3}$ P. Benetti, ${ }^{4,5}$ L. Cardani, ${ }^{2,3}$ N. Casali, ${ }^{6,7}$ D. Chiesa, ${ }^{8,9}$ \\ M. Clemenza, ${ }^{8,9}$ I. Dafinei, ${ }^{3}$ S. Di Domizio, ${ }^{10,11}$ F. Ferroni, ${ }^{2,3}$ A. Giachero, ${ }^{8,9}$ L. Gironi, ${ }^{8,9}$ \\ A. Giuliani, ${ }^{12}$ C. Gotti, ${ }^{9}$ M. Maino, ${ }^{8,9}$ S. Nagorny, ${ }^{7,13}$ S. Nisi, ${ }^{7}$ C. Nones, ${ }^{14}$ F. Orio, ${ }^{3}$ \\ L. Pattavina, ${ }^{7}$ G. Pessina, ${ }^{9}$ G. Piperno, ${ }^{2,3}$ S. Pirro, ${ }^{7}$ E. Previtali, ${ }^{9}$ C. Rusconi, ${ }^{9}$ \\ M. Tenconi, ${ }^{12}$ C. Tomei, ${ }^{3}$ and $M$. Vignati ${ }^{3}$
}

${ }^{1}$ Lawrence Berkeley National Laboratory, Berkeley, CA 94720, USA

${ }^{2}$ Dipartimento di Fisica, Sapienza Università di Roma, 00185 Roma, Italy

${ }^{3}$ INFN, Sezione di Roma 1, 00185 Roma, Italy

${ }^{4}$ Dipartimento di Chimica, Università di Pavia, 27100 Pavia, Italy

${ }^{5}$ INFN, Sezione di Pavia, 27100 Pavia, Italy

${ }^{6}$ Dipartimento di Fisica, Università degli Studi dell'Aquila, 67100 L’Aquila, Italy

${ }^{7}$ INFN, Laboratori Nazionali del Gran Sasso, 67010 Assergi, Italy

${ }^{8}$ Dipartimento di Fisica, Università di Milano Bicocca, 20126 Milano, Italy

${ }^{9}$ INFN, Sezione di Milano Bicocca, 20126 Milano, Italy

${ }^{10}$ INFN, Sezione di Genova, 16146 Genova, Italy

${ }^{11}$ Dipartimento di Fisica, Università di Genova, 16126 Genova, Italy

${ }^{12}$ Centre de Spectrométrie Nucléaire et de Spectrométrie de Masse, 91405 Orsay, France

${ }^{13}$ Institute for Nuclear Research, MSP, Kyiv 03680, Ukraine

${ }^{14}$ CEA, Irfu, SPP Centre de Saclay, 91191 Gif-sur-Yvette, France

Correspondence should be addressed to S. Pirro; stefano.pirro@lngs.infn.it

Received 27 July 2013; Accepted 30 September 2013

Academic Editor: Vincenzo Flaminio

Copyright (C) 2013 J. W. Beeman et al. This is an open access article distributed under the Creative Commons Attribution License, which permits unrestricted use, distribution, and reproduction in any medium, provided the original work is properly cited.

In the field of fundamental particle physics, the neutrino has become more and more important in the last few years, since the discovery of its mass. In particular, the ultimate nature of the neutrino (if it is a Dirac or a Majorana particle) plays a crucial role not only in neutrino physics, but also in the overall framework of fundamental particle interactions and in cosmology. The only way to disentangle its ultimate nature is to search for the neutrinoless double beta decay. The idea of LUCIFER is to combine the bolometric technique proposed for the CUORE experiment with the bolometric light detection technique used in cryogenic dark matter experiments. The bolometric technique allows an extremely good energy resolution while its combination with the scintillation detection offers an ultimate tool for background rejection. The goal of LUCIFER is not only to build a background-free small-scale experiment but also to directly prove the potentiality of this technique. Preliminary tests on several detectors containing different interesting DBD emitters have clearly demonstrated the excellent background rejection capabilities that arise from the simultaneous, independent, double readout of heat and scintillation light.

\section{State of the Art}

The double beta transition, which is the rarest nuclear weak process, takes place between two even-even isobars, when the decay to the intermediate nucleus is energetically forbidden due to the pairing interaction, which opens a gap between the even-even and the odd-odd mass parabolas in a given isobaric chain. The two-neutrino decay conserves the lepton number and was originally proposed by Goeppert-Mayer in 1935 [1]. It is a second-order weak process-that explains its 
low rate - and it has been observed for a dozen of nuclei, with lifetimes in the range of $10^{18}-10^{22} \mathrm{y}$ [2].

Besides the two-neutrino decay, a much more intriguing process, the so-called neutrinoless double beta decay $0 v$-DBD [3-6], was proposed by Furry [7] shortly after the Majorana theory of the neutrino [8]. In this case, the simultaneous transformation of two neutrons into two protons is accompanied by the emission of two electrons and nothing else. The main feature of $0 v$-DBD is just the violation of the lepton number. In the modern (standard model) perspective, this is as important as the violation of the baryon number. In full generality, we can imagine this process as a mechanism capable of creating electrons in a nuclear transition.

It is remarkable that $0 \nu$-DBD is not necessarily due to the exchange of Majorana neutrinos (mass mechanism) as a leading contribution, even though its detection would demonstrate that neutrinos are self-conjugate particles [9]. In spite of the wide variety of mechanisms possibly inducing the $0 \nu$-DBD, after the discovery of neutrino flavor oscillations (which prove that neutrinos are massive particles), the mass mechanism occupies a special place. It relates neatly the $0 v$ DBD to important parameters of neutrino physics, fixes clear experimental targets, and provides a clue to compare on equal footing experiments which present considerable differences from the methodological and technological points of view. In fact, the lifetime of the $0 v$-DBD decay is related to the so-called effective Majorana neutrino mass $\left\langle m_{\nu}\right\rangle$, a crucial parameter that contains the three neutrino masses $m_{1}, m_{2}$, and $m_{3}$, the elements of the first row of the neutrino mixing matrix, and the unknown CP-violating Majorana phases, which make the cancellation of terms possible; $\left\langle m_{\nu}\right\rangle$ could be smaller than any of the three neutrino masses.

Majorana mass and weak isospin selection rules make it possible to find a natural explanation to the smallness of neutrino mass. The pattern of neutrino masses and mixing admits an elegant solution, the so-called see-saw mechanism. At the same time, the only viable explanation of the matterantimatter asymmetry available today, the justification for our very existence, is based on the leptogenesis, which again requires a Majorana nature for neutrinos [10]. This well motivates why $0 \nu$-DBD plays a central role in particle physics and cosmology.

Thanks to the information we have from oscillations and assuming the standard three active neutrino scenarios, it is useful to express the effective Majorana neutrino mass $\left\langle m_{\nu}\right\rangle$ in terms of three unknown quantities: the mass scale, represented by the mass of the lightest neutrino $m_{\min }$, and the two Majorana phases. It is then common to distinguish three mass patterns: normal hierarchy, where $m_{1}<m_{2}<m_{3}$, inverted hierarchy where, $m_{3}<m_{1}<m_{2}$, and the quasidegenerate spectrum, where the differences between the masses are small with respect to their absolute values. We ignore the neutrino mass ordering at the moment, and the $0 v$ DBD has the potential to provide this essential information.

In the standard interpretation of $0 \nu$-DBD in terms of mass mechanism, three challenges are in front of the experimentalists who study this process. The first one consists in scrutinizing the much debated ${ }^{76} \mathrm{Ge}$ claim [11]; recent experimental results $[12,13]$ have practically accomplished this task.
The second one consists in approaching and then covering the inverted hierarchy region of the neutrino mass pattern. The third and ultimate goal is to explore the direct hierarchy region.

While scrutinizing the ${ }^{76} \mathrm{Ge}$ claim can be done in principle with only $\sim 10 \mathrm{~kg}$ of isotope, we need typically 1 ton of isotope mass in order to explore the inverted hierarchy region, just to accumulate a few signal counts. The direct hierarchy region seems for the moment out of the reach of the present technologies, since one would need sources of the order of $1 \mathrm{Mmol}$ (typically 100 tons).

Just having a large source is not enough; in order to appreciate such tiny signal rates, the experimentalists are obliged to operate in conditions of almost zero background. Acceptable background rates are of the order of 1-10 counts/y/ton if the goal is just to approach or touch the inverted hierarchy region, whereas one needs at least one order of magnitude lower values to explore it fully, around or even less than 1 count/y/ton.

The experimental strategy pursued to investigate the $0 v$ DBD consists of the development of a proper nuclear detector, with the purpose of revealing the two emitted electrons in real time and collecting their sum energy spectrum as minimal information. Indeed, the shape of the two electron sum energy spectrum enables us to distinguish between the two discussed decay modes. In case of $2 v$-DBD, this spectrum is a continuum between 0 and the $Q_{\beta \beta}$-value with a maximum around $1 / 3 \cdot Q_{\beta \beta}$. For $0 \nu$-DBD, the spectrum is just a peak at the transition energy, enlarged only by the finite energy resolution of the detector.

The ideal desirable features of this nuclear detector are: (i) high energy resolution, as a peak must be identified over an almost flat background; (ii) large source, in order to monitor many candidate nuclides; and (iii) low background, which requires underground detector operation to shield from cosmic rays, very radiopure materials, well-designed passive and/or active shielding against local environmental radioactivity and possibly event tracking andtopology capability. This last point is useful not only to reject the background but also to provide additional kinematical information on the emitted electrons [14]. Typically, the listed features cannot be met simultaneously in a single detection method.

The searches for $0 v$-DBD can be further classified into two main categories: the so-called calorimetric technique, in which the source is embedded in the detector itself and which provides extremely high efficiency, and the external-source approach, in which source and detector are two separate systems, allowing excellent event reconstruction.

Which are the best isotopes to search for $0 v$-DBD? Experimental practice shows that the following three factors weigh the most in the design of an experiment: (i) the $Q_{\beta \beta^{-}}$ value; (ii) the isotopic abundance together with the ease of enrichment; and, last but not least, (iii) the compatibility with an appropriate detection technique. The $Q_{\beta \beta}$-value is probably the most important criterion. It influences both the phase space and the background. On the basis of $Q_{\beta \beta}$-value selection, at the moment there are only 9 experimentally relevant isotopes. The transition energies of all these isotopes are larger than $2.4 \mathrm{MeV}$, with the important exception of 
${ }^{76} \mathrm{Ge}\left(Q_{\beta \beta}\right.$-value $\left.=2.039 \mathrm{MeV}\right)$. There are two important energy markers in terms of background which have to be compared with the $Q_{\beta \beta}$-value: (i) the $2615 \mathrm{keV}$ line represents the endpoint of the bulk of the natural gamma radioactivity; (ii) the $3270 \mathrm{keV}$ line represents the $Q$-value of the ${ }^{214} \mathrm{Bi} \beta$ decay, which, among the ${ }^{222} \mathrm{Rn}$ daughters, is the one releasing the highest-energy $\beta$ 's and $\gamma$ 's. The 9 candidates are divided according to these two markers into three groups of three isotopes each.

The first group $\left({ }^{76} \mathrm{Ge},{ }^{130} \mathrm{Te}\right.$, and $\left.{ }^{136} \mathrm{Xe}\right)$ has to cope with some gamma background and with the radon-induced one; however, very sensitive experiments can be performed with these nuclides since they are particularly suitable to be studied with a calorimetric approach. Germanium semiconductor diodes are excellent devices for the isotope ${ }^{76} \mathrm{Ge}$ $[11,13] ; \mathrm{TeO}_{2}$ bolometers are at the forefront due to their high content of ${ }^{130} \mathrm{Te}$ [15], while the last of these three isotopes can be easily embedded in gaseous or liquid TPC $[16,17]$ or in large volumes of a liquid scintillator [12].

The second group $\left({ }^{82} \mathrm{Se},{ }^{100} \mathrm{Mo}\right.$, and $\left.{ }^{116} \mathrm{Cd}\right)$ is out of the reach of the bulk of the gamma environmental background; as shown in the next section, they belong to the realm of the scintillating bolometers, which are almost perfectly suited to investigate these isotopes. LUCIFER's experimental results described below show that at least two of them, namely, ${ }^{82} \mathrm{Se}$ and ${ }^{100} \mathrm{Mo}$, can be studied with high sensitivity.

The candidates of the third group $\left({ }^{48} \mathrm{Ca},{ }^{96} \mathrm{Zr}\right.$, and ${ }^{150} \mathrm{Nd}$ ) are in the best position to realize a background-free experiment. For a sort of conspiracy of nature, however, these three golden-plated elements cannot be enriched at low cost and high throughput.

We are now (September 2013) at a turning point in the experimental search for $0 v$-DBD decay. The ${ }^{76} \mathrm{Ge}$ claim is strongly disfavored after the results provided by EXO-200 [16], KamLAND-Zen [12], and especially GERDA (phase I) [13], which investigates the same isotope as the claim and is therefore free from the systematics induced by the calculation of the nuclear matrix elements. The follow-up of these searches and others which are in an advanced construction phase (CUORE [15] and SNO+ [18]) promises to go well below $0.2 \mathrm{eV}$. Therefore, the first of the three aforementioned challenges is very close to be achieved, and it will be in the near future.

However, there is no univocal strategy to deal with the second challenge, namely, to explore deeply the inverted hierarchy band of the neutrino mass pattern. Searches in preparation can only approach the onset of this region at $\left\langle m_{\nu}\right\rangle \sim$ $0.05 \mathrm{eV}$.

The place of LUCIFER in this context is very clear. This experiment does not foresee at the moment a sensitivity comparable to those of the most advanced experiments. In fact, LUCIFER is essentially an R\&D activity, aiming at building a technology demonstrator at the $10-15 \mathrm{~kg}$ scale. However, it will face and hopefully solve all the issues related to a final search. Therefore, the LUCIFER results will have a major impact on the investigation for $0 \nu$-DBD. All the experimental indications collected so far and reported in detail in this work show not only that the LUCIFER technology is viable, but also that the experiments which will adopt it are among the best positioned to achieve a background of the order of 1 count/y/ton, combined with the high energy resolution of the bolometric technique, the sensitive mass scalability guaranteed by the detector modular structure, and the high efficiency property of the calorimetric approach. They would have therefore all the numbers to achieve the second challenge in the search for $0 \nu$-DBD decay.

\section{Bolometers and Scintillating Bolometers}

Cryogenic particle detectors (see, e.g., [19]) have been used for many years for the study of rare events such as $0 v$-DBD and dark matter (DM) searches.

A cryogenic bolometer is simply made of a suitable absorber and a thermometer coupled with it. When a particle releases energy within the absorber, a tiny temperature rise can be measured with an appropriate thermometer, provided that the heat capacity of the absorber is reasonably small. This can be fulfilled even with a multi-kg absorber [20] provided that it is made of a dielectric crystal kept at cryogenic temperatures of the order of tens of $\mathrm{mK}$.

Bolometers offer a wide choice of absorber materials, and at the same time, they are able to achieve an energy resolution competitive with that of Ge diodes (viz. of the order of $5 \mathrm{keV}$ FWHM at $3 \mathrm{MeV}$ ). The freedom in the choice of the absorber provides the unique opportunity of selecting the $\mathrm{DBD}$ isotope without the limitations usually induced by the experimental technique (e.g., with semiconductor detectors).

The CUORE experiment represents the most advanced stage in the use of bolometers for $0 v$-DBD search. CUORE will consist of an array of 988 crystals for a total mass of $\simeq 1$ ton of $\mathrm{TeO}_{2}$. Its data taking is expected to begin in 2015 .

Unlike other solid-state devices, however, bolometers are not ionization detectors but phonon detectors. As a consequence, they are almost equally sensitive to any kind of particle, despite the way energy is released. In other words electrons, $\alpha$ particles, and nuclear recoils-depositing the same amount of energy in the detector-produce a pulse with the same amplitude and shape. The Cuoricino [21] data demonstrated that the background in the region of interest is dominated by radioactive contaminations on the surfaces facing the detectors. $\alpha$ particles produced by these contaminants can lose a fraction of their energy in the host material and the rest in the detector, thus producing a flat background from the energy of the decay (several $\mathrm{MeV}$ ) down to the $0 v$ DBD region [22].

The background induced by surface $\alpha$-emitters represents, in fact, the limiting background in the region of interest for this technique [23].

Scintillating bolometers, developed in recent years for DM searches $[24,25]$ and proposed also for $0 \nu$-DBD surveys [26], allow overcoming this disadvantage by providing the possibility to distinguish $\alpha$ interactions (background only) from $\beta / \gamma$ interactions (background and signal). A scintillating bolometer is obtained by coupling a scintillating crystal, operating as a cryogenic bolometer (as described above), to a proper light detector (see Section 5). 
When a particle traverses the scintillating crystal, a large fraction of the deposited energy is converted into heat (thus inducing a temperature rise), while the remaining small fraction is spent to produce scintillation light.

The interesting feature of scintillating bolometers is that the ratio between the two signals (light/heat) depends on the particle mass and charge. Particles like $\beta$ 's and $\gamma$ 's have the same light emission (conventionally referred to as the light yield (LY), i.e., the fraction of particle energy emitted in photons) which is typically different from the light emission of $\alpha$ particles or neutrons. Consequently, the simultaneous readout of the heat and light signals allows particle discrimination.

If the scintillating crystal contains a DBD candidate, the $0 v$-DBD signal (i.e., the energy deposition produced by the two electrons emitted after the decay) can be distinguished from an $\alpha$ signal, and only $\beta$ 's and $\gamma$ 's can give a sizable contribution to the background that limits the experimental sensitivity. The feasibility of this technique is today widely proven. Scintillating bolometers containing $\mathrm{Ca}, \mathrm{Mo}, \mathrm{Cd}$, and Se have been successfully tested, coupled to a thin Ge wafer operating as bolometer for the light readout [27-30]. Presently, those that look most promising for a $0 v$-DBD large scale experiment are $\mathrm{ZnSe}$ and $\mathrm{ZnMoO}_{4}$.

\section{The LUCIFER Layout}

The LUCIFER setup will consist of an array of individual single module detectors, arranged in a tower-like structure, as schematized in Figure 1. The single module (see Section 6) will consist of a cylindrical crystal of $45 \mathrm{~mm}$ diameter and $55 \mathrm{~mm}$ height, equipped with a Ge-crystal light detector (see Section 5). The crystal is fastened by means of four S-shaped Teflon pieces fixed to the two cylindrical $\mathrm{Cu}$ frames. The frames are held together through two Cu columns. The crystal is surrounded (without being in thermal contact) with a reflecting plastic foil (3M VM2002) in order to increase the light collection. Individual single modules allow us to test in advance each single detector before assembling the entire tower experiment.

The tower will be installed in the same dilution cryostat that hosted the Cuoricino experiment. The cryogenic layout and the readout electronics (see Section 4) are based on the experience gained in the Cuoricino experiment and implemented in the CUORE project.

The key point of the experiment will be the crystal growth made with enriched isotope, as discussed in Section 7. With the enriched material being extremely expensive, the losses during the various stages of the production have to be minimized. This R\&D is still ongoing and could slightly change some parameter of the layout (number of crystals and their final dimensions).

The evaluation of the background and of the sensitivity foreseen for this layout, as explained in Section 9, takes advantage of the Cuoricino experiment that was able to characterize and simulate the various dangerous contaminants present in the cryogenic facility. As explained in Section 1, the main source of background arises from ${ }^{226} \mathrm{Ra}$ that will produce ${ }^{214} \mathrm{Bi}$ whose rare, but high, energies $\gamma$ s can travel

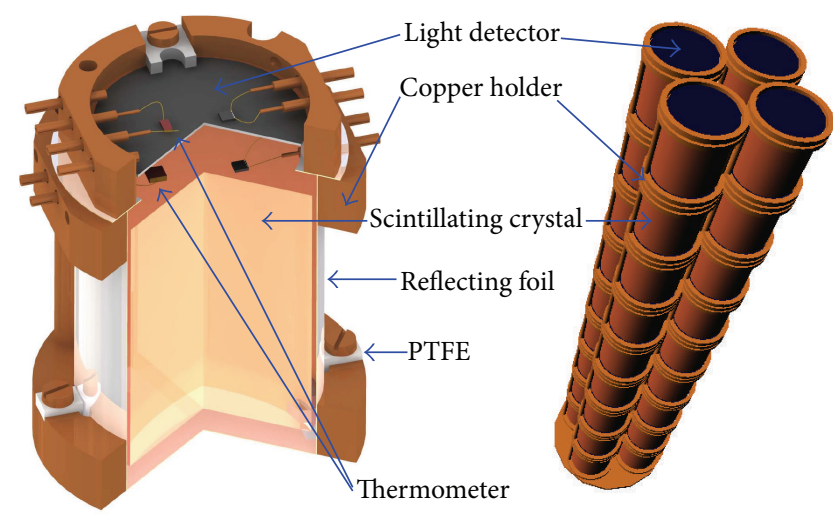

FIGURE 1: LUCIFER layout. Left: single module detector consisting of the scintillating bolometer and the light detector. Right: 9-floor tower constituted by 36 single modules.

enough to reach the tower. With respect to ${ }^{228} \mathrm{Th}$, producing the ${ }^{208} \mathrm{Tl}$ whose decay represents the real "nightmare" for all the DBD experiments, the situation is rather different. Even if the $Q$-value of the ${ }^{208} \mathrm{Tl} \beta$-decay is the highest among the natural radioactive chains, in order to produce background above $2615 \mathrm{keV}$, the contamination must be present in the proximity of the detectors, so that summing effects can take place. For the LUCIFER project, all the materials (Cu holders, and thermal shields of the cryostat) within the first $5 \mathrm{~cm}$ from the detectors will be replaced with new ones that will be produced with already selected and tested $\mathrm{Cu}$, whose ${ }^{228} \mathrm{Tl}$ activity is found to be negligible.

\section{The Electronic System}

The thermometers used in LUCIFER are thermistors (see Section 8). These devices show a resistivity that changes exponentially with the temperature being below $100 \mathrm{mK}$. The readout scheme is, in principle, very simple; the thermistor is polarized by a simple bias circuit, consisting of a voltage generator closed on two load resistors; when a particle releases energy in the absorber, the temperature rises and the resistance of the thermistors changes, thus producing a measurable voltage drop. Given that the study of rare events such as $0 \nu$-DBD is performed over a long period of time, the overall stability of the readout system plays a crucial role.

To fully exploit this stringent requirement and to match the DAQ dynamic range, a dedicated system has been developed, as schematized in Figure 2; the bolometer, Bol, is coupled with the thermistor, $R_{B}$. It is inside the fridge, at a temperature between 10 and $20 \mathrm{mK}$.

Signals from bolometers (depending on their mass) show a signal bandwidth from a few tens of $\mathrm{Hz}$ up to a few hundreds of $\mathrm{Hz}$. In case of relatively fast detector response (e.g., light detectors; see next section), a unity gain buffer and the load resistors are located inside the fridge [31-34], at a larger temperature this time, $120 \mathrm{~K}$. This is the box Option in Figure 2, which is close to the detectors (a few tens of $\mathrm{cm}$ in terms of signal wire), to minimize parasitic capacitance. The box 


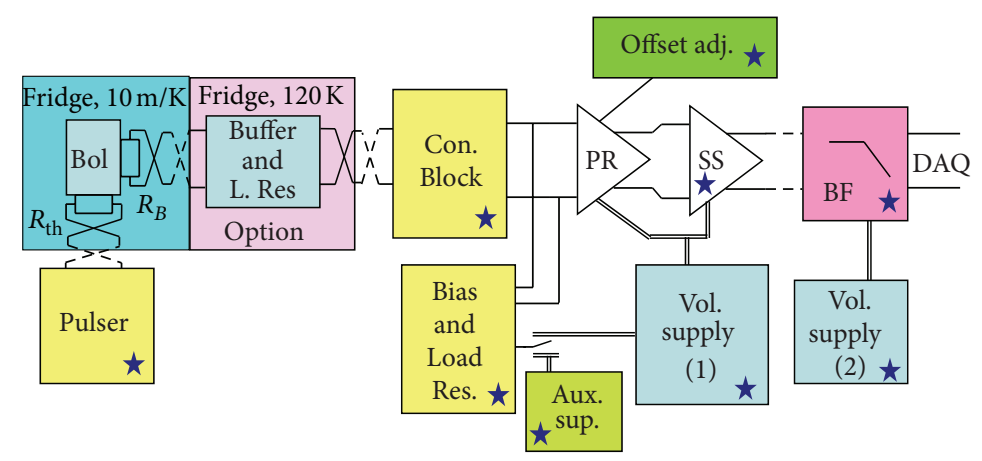

FIGURE 2: Schematic diagram of the electronics system of LUCIFER. Stars on the blocks denote the presence of remote programmability.

Option is not present for the readout of the slow (massive) detectors, and, in this case, the thermistor $R_{B}$ is connected directly to the box Con. Block, at room temperature, through a small thermal conductance electrical link [35], and, in this case also, the load resistors are at room temperature (Bias and Load Res.). All the electrical connections of the detectors consist of twisted pairs of wires, so that all the possible sources of common mode disturbances and crosstalk between nearby channels are minimized [36, 37].

The Con. Block of Figure 2 allows us to connect the preamplifier, $\mathrm{PR}$, to the detector, to the ground, or to a test pulse. This block, together with Bias and Load Res., that can switch between 2 different sets of load resistances and set the level and polarity of the detector bias voltage, is exploited for DC characterization of the detectors to optimize their working points, detector by detector [38]. The detector load resistances may have an impact on the thermal and low-frequency parallel noise. The minimization of their low-frequency noise, otherwise proportional to the square of the applied voltage, has been extensively studied [39] and addressed. Other noise sources originate from the PR. Also in this case, the input transistors, a pair of JFETs, have been selected with a semicustom approach [40]. Moreover, in order to decrease the thermal drift of the preamplifiers, a special circuit was developed $[41,42]$ that ensures a maximum drift of the order of a fraction of $\mu \mathrm{V} /{ }^{\circ} \mathrm{C}$.

Remote programmability [37] is available for many parts of the electronic system, marked with a star in Figure 2. DC coupling is necessary not only for what concerns signal analysis but also for detector monitoring.

The second-stage amplifier (SS) buffers the outputs of PR with a programmable gain. The outputs of SS are connected to the DAQ system after the antialiasing filter BF that consists of a 6-pole-roll-off Thomson or Bessel filter. The antialising filter has a few programmable frequency bandwidths [43]. The blocks before the BF are all located on the top of the cryostat, just a few $\mathrm{cm}$ away from it. The BF is instead located close to the $\mathrm{DAQ}$, in a remote location, so as to reject every possible disturbance from the long connecting links.

The power supply of LUCIFER, as well as parts of the electronics system previously described, is an upgraded version of [44]. The DC input to the power supply is derived from a 2 -stage AC/DC system based on an AC (main line)/48 V DC and a $48 \mathrm{~V} / \pm 11 \mathrm{~V} / \pm 6 \mathrm{~V} / 6 \mathrm{~V}$.

\section{Light Detectors}

The first light/heat measurement was performed with a thermal bolometer and a silicon photodiode in 1992 [45] but was no longer pursued due to the difficulties of running a "standard photodevice" at cryogenic temperatures. The use of a bolometer as a light detector (LD) was first developed in 1997 [46] and further optimized for DM searches [24, 25] after a few years. The first developments for $0 \nu$-DBD searches began in 2006 [47].

The LUCIFER light detectors consist of thin germanium slabs, operating as bolometers, facing the main scintillating crystal. The choice of such material is driven by several considerations. Being opaque semiconductors, they are sensitive over an extremely wide range of photon wavelengths and, moreover, satisfy the very stringent radiopurity requirements of rare event searches. Their overall quantum efficiency can be as good as the one of photodiodes. To further increase the light collection, a $\mathrm{SiO}_{2}$ dark layer [48] is deposited on the surface of the Ge crystal that faces the bolometer. Each LUCIFER light detector consists of a disk-shaped pure Ge crystal $(\varnothing=44 \mathrm{~mm}$, thickness $=180 \mu \mathrm{m})$ grown using Czochralski technique and purchased from Umicore. The temperature sensor is a neutron transmutation doped (NTD) Ge thermistor (see Section 8).

The scintillation photons, as well as other particles, interact in the LD and deposit their energy within its volume. The energy deposition is eventually converted into phonons, causing a temperature rise in the system and, in particular, in the NTD thermistor.

To allow proper calibration of the signal, a ${ }^{55} \mathrm{Fe}$ source, producing two X-rays at 5.9 and $6.5 \mathrm{keV}$, is faced to the light detector. The choice of such low-energy calibration lines is due to the fact that light signals produced in scintillating bolometers have typical energies of the order of $\sim 10 \mathrm{keV}$.

As shown in [49], LUCIFER light detector performances have a clear dependence on the applied bias current, both in terms of energy resolution and signal time development (see Figure 3). As will be shown in Section 6.2, a very efficient particle discrimination can be obtained using the time development of the scintillation signal. Therefore, very often, the working point of the $\mathrm{LD}$ is chosen as a compromise between good energy resolution and fast detector response, obtained by tuning the polarization current. 


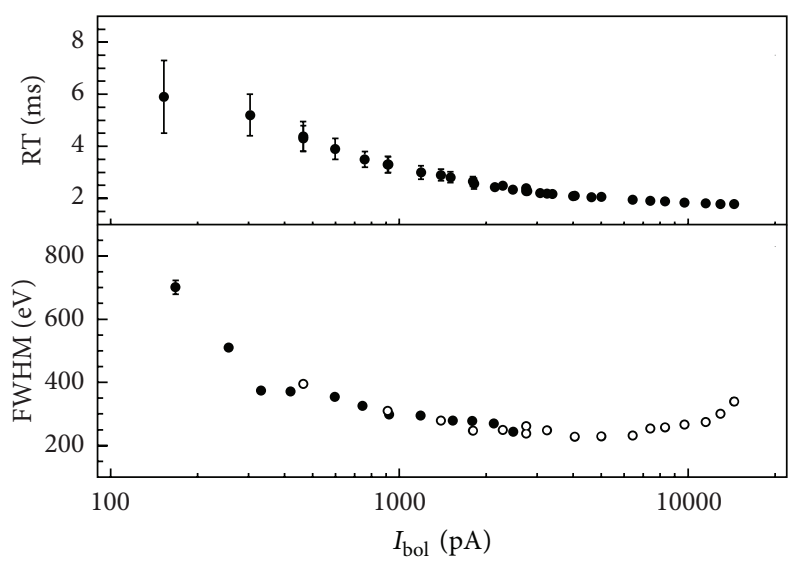

(a)

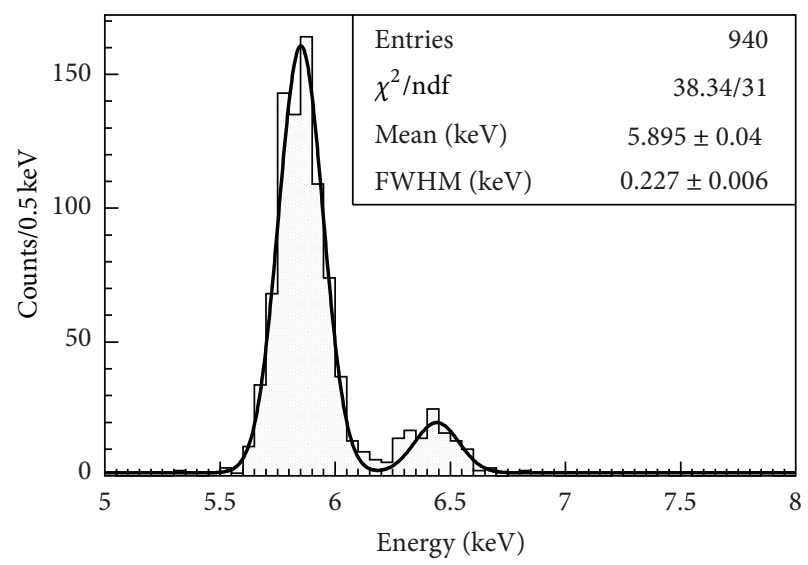

(b)

FIGURE 3: (a) LD performances (rise time and FWHM energy resolution) as a function of the polarization current, using load resistors of $11 \mathrm{G} \Omega$ (black dots) and $2 \mathrm{G} \Omega$ (open circles). (b) calibration spectrum of the detector, using a ${ }^{55} \mathrm{Fe} X$-ray source.

\section{Bolometric Investigation of the Crystals}

6.1. Experimental Setup. As explained in Section 2, bolometers offer a wide choice in terms of the absorber material. An extensive R\&D activity was carried out over these last years in order to understand the features of the various crystals, and several prototypes of different masses and production lines were investigated. All the analyzed crystals were operated as bolometers in the deep underground facilities of Laboratori Nazionali del Gran Sasso, Italy.

The detectors were installed in a ${ }^{3} \mathrm{He} /{ }^{4} \mathrm{He}$ dilution refrigerator (operating at about $10 \mathrm{mK}$ ) properly shielded from external radiations ( $\beta$ 's $/ \gamma$ 's and neutrons) by means of lead and polyethylene shields, inside a Faraday cage continuously flushed with nitrogen. The experimental setup consists of a copper structure for the housing of the crystals and light detectors. In order to avoid the noise induced by the vibration of the cryogenic facility, the structure is mechanically decoupled [50] from the cryostat by using a two stage damping system [51]. The thermal stability of the holder (whose variations are within a few tens of $\mu \mathrm{K}$ ) is ensured by a completely custom proportional, integrative, and derivative (PID) feedback loop [52]. In order to further increase the stability of each single detector, a resistor of $100 \div 300 \mathrm{k} \Omega$, realized with a heavily doped meander on a $3.5 \mathrm{~mm}^{3}$ silicon chip, is attached to each bolometer and acts as a heater to stabilize the gain of the detector [53] by injecting "monochromatic" voltage pulses $[54,55]$ across the heater.

The heat and light signals produced by interacting particles into the absorbers are transformed into voltage pulses by NTD thermistors; then the signals are amplified and fed into an 18 bit NI-6284 PXI ADC unit. Software triggers ensure that every thermistor pulse is recorded with a $0.5 \div 2 \mathrm{kHz}$ sampling rate over a $0.25 \div 5 \mathrm{~s}$ duration (depending on the crystal). Moreover, when a trigger is generated by the main crystal, the corresponding waveform from the LD is recorded, irrespectively of its trigger. The amplitude and the shape of the voltage pulse are determined by the offline analysis that makes use of the optimum filter technique $[56,57]$. The signal amplitudes are computed as the maximum of the filtered pulse. The amplitude of the light signal is estimated from the value of the filtered waveform at a fixed time delay with respect to the signal of the bolometer, as described in detail in [58].

The energy calibration of the crystals is performed using removable $\gamma$ sources placed outside the cryostat. The heat channel is calibrated, attributing to each identified $\gamma$-peak the nominal energy of the line, as if all the energy is converted into heat. Consequently, this calibration does not provide an absolute evaluation of the heat deposited in the crystal. It has to be remarked that in scintillating bolometers, the energy scale of $\gamma / \beta$ and $\alpha$ particles is different. This is simply induced by the different scintillation yield; in standard scintillators, for example, $\gamma / \beta$ particles scintillate more than $\alpha$ 's with the same impinging energy. This energy, therefore, "escapes from the crystal" and, as a consequence, implies a different energy scale for a different type of particles [29]. For this reason, the energy scale is, usually, labelled as $\mathrm{keV}_{\text {ee }}$ (electron equivalent), meaning that the energy scale is calibrated on $\gamma / \beta$ particles. The energy difference between the two scales is normally at the few \% level, but for $\mathrm{ZnSe}$ it can reach larger values [30] that cannot be explained in terms of escaping light.

6.2. $\mathrm{Zn}^{82} \mathrm{Se}$. Because of its high content of Se (56\%), as well as its good bolometric and scintillating properties, $\mathrm{ZnSe}$ has always been an interesting candidate for the search of the $0 \nu$ $\mathrm{DBD}$ of ${ }^{82} \mathrm{Se}\left(Q_{\beta \beta}=2995 \mathrm{keV}\right)$.

The largest $\mathrm{ZnSe}$ bolometer ever realized, a $431 \mathrm{~g}$ crystal grown at ISMA, Ukraine, was recently characterized in terms of energy resolution, internal contaminations, and particle identification capabilities.

The FWHM energy resolution was found to be $13.4 \pm$ $1.0 \mathrm{keV}$ at $1461 \mathrm{keV}$ and $16.3 \pm 1.5 \mathrm{keV}$ at $2615 \mathrm{keV}$. However, starting from the consideration that light and heat are two correlated estimates of the particle energy, we developed an 


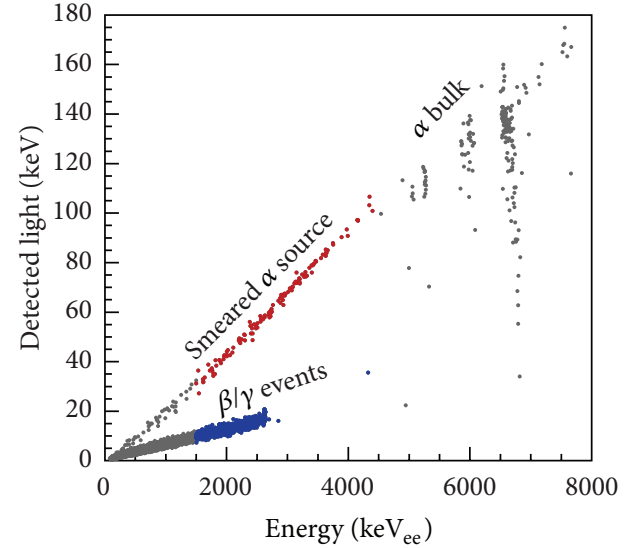

(a)

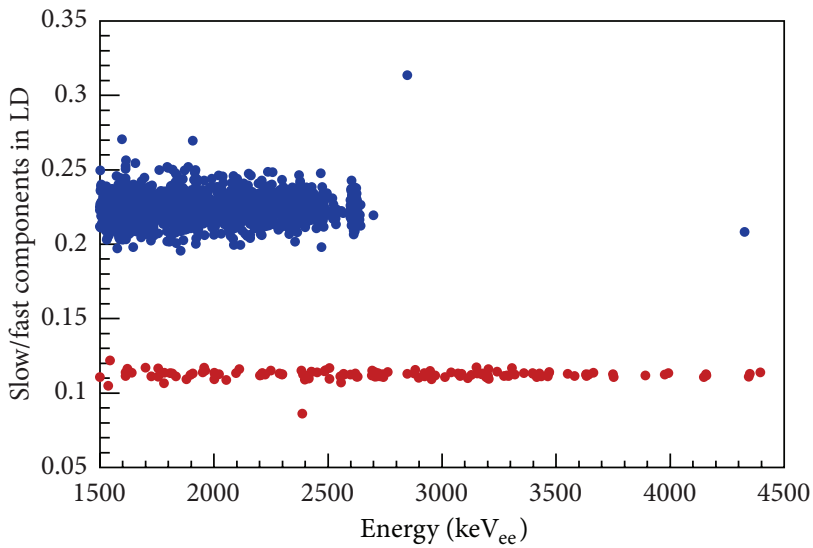

(b)

Figure 4: Particle discrimination in ZnSe. The light emitted by the ZnSe crystal (a) and the shape of the same light pulses (b) are reported as a function of the energy released in the $\mathrm{ZnSe}$ bolometer. A ${ }^{228} \mathrm{Th} \gamma$-source was used to produce $\beta / \gamma$ events (blue), up to $2615 \mathrm{keV}\left({ }^{208} \mathrm{Tl}\right)$, while a smeared $\alpha$ source was placed under the crystal to provide a continuum of $\alpha$ 's extending to lower energies (red). The (heat channel) $x$-axis is (energy) calibrated using the most intense $\gamma$-peaks. The extremely different energy scale between $\alpha$ and $\beta / \gamma$ events can be observed, as discussed in Section 6.1. The blue and red points of the upper plot are determined by a cut on the bottom plot.

analysis algorithm that allowed us to considerably improve the energy resolution by removing this correlation. The application of the algorithm, whose details are described in [59], resulted in a FWHM energy resolution of $12.2 \pm 0.8 \mathrm{keV}$ at $1461 \mathrm{keV}$ and $13.4 \pm 1.3 \mathrm{keV}$ at $2615 \mathrm{keV}$.

Many runs were performed in order to assess the scintillation properties of $\mathrm{ZnSe}$ at cryogenic temperatures. In Figure 4(a), the detected scintillation light is reported as a function of the heat released in the crystals. In order to study the discrimination power between $\beta / \gamma$ and $\alpha$ events, a uranium $\alpha$ source covered with a thin mylar foil was placed close to the ZnSe surface. The mylar foil degrades the energy of the emitted $\alpha$ 's, producing a continuum at low energy (red dots in the plot). Using a ${ }^{228} \mathrm{Th} \gamma$-source, (outside the cryostat) and the "internal" $\alpha$ source, we were able to investigate the discrimination potential in the DBD energy region.

Looking at Figure 4(a), we observed that the events produced by the $\gamma$-source (blue) lie in a different region with respect to $\alpha$ particles with the same energy. The LY of $\beta / \gamma$ events resulted in $\mathrm{LY}_{\beta / \gamma}=6.416 \pm 0.008 \mathrm{keV} / \mathrm{MeV}$, irrespective of the energy deposit. The LY of $\alpha$ particles was studied separately for surface and internal $\alpha$ contaminations, resulting in $\mathrm{LY}_{\alpha}^{\text {surf }}=29.70 \pm 0.17 \mathrm{keV} / \mathrm{MeV}$ and $\mathrm{LY}_{\alpha}^{\text {bulk }}=$ $26.62 \pm 0.86 \mathrm{keV} / \mathrm{MeV}$. Several tests showed that the difference in the LY of bulk/surface events cannot be ascribed to self-absorption in the crystal or to energy dependence of the effects. Therefore, the origin of this behavior must reside in an effectively larger light production on the crystal surface or in nonuniformities in the light collection.

The larger LY of $\alpha$ events with respect to $\beta / \gamma$ in $\mathrm{ZnSe}$ crystals is not yet understood and, in principle, could affect the discrimination capability. A poor efficient light collection for $\alpha$ particles, indeed, can generate $\alpha$ event leaking in the $\beta / \gamma$ band, increasing the background in the region of interest (see Figure 4(a)).
In order to overcome this problem, we developed an analysis algorithm which is sensitive to the difference in the pulse shape of $\alpha$ 's and $\beta / \gamma$ 's (see details in [59]). Each pulse was fitted using a model that takes into account the development of the phonon signals, the thermistor and electronics response, and the scintillation process, which cannot be considered instantaneous. Applying this algorithm to the heat and light pulses of $\mathrm{ZnSe}$, we discovered that a poor particle discrimination can be obtained using the heat channel alone. The shape of the light pulses, on the contrary, is very sensitive to the type of interacting particles (see Figure 4(b)).

Thanks to the pulse shape discrimination, it is possible to identify and reject the $\alpha$ interaction with very high efficiency, thus strongly reducing the background in the energy region of interest.

Finally, we performed a 524-hour background run to investigate the internal contaminations of the ZnSe crystal.

The study of the $\alpha$ spectrum highlighted a contamination of $17.2 \pm 4.6 \mu \mathrm{Bq} / \mathrm{kg}$ in ${ }^{232} \mathrm{Th}$ and of $24.6 \pm 5.5 \mu \mathrm{Bq} / \mathrm{kg}$ in ${ }^{238} \mathrm{U}$, both in equilibrium with their daughters.

The $\beta / \gamma$ energy spectrum of this measurement, obtained using cuts on the LY and pulse shape, is reported in Figure 5. Besides the ${ }^{40} \mathrm{~K}$ and ${ }^{208} \mathrm{Tl}$ peaks, due to the natural contaminations of the environment, only contaminations in ${ }^{75} \mathrm{Se}$ $\left(T_{1 / 2}=119.8 \mathrm{~d}, Q\right.$-value $\left.=863.6 \mathrm{keV}\right)$ and ${ }^{65} \mathrm{Zn}\left(T_{1 / 2}=244 \mathrm{~d}\right.$, $Q$-value $=1359.9 \mathrm{keV}$ ) were found. The presence of these isotopes, due to the activation of ${ }^{74} \mathrm{Se}$ and ${ }^{64} \mathrm{Zn}$, respectively, does not affect the background in the DBD region because of their short half-lives and low $Q$-values.

Above the $2615 \mathrm{keV} \gamma$-line, a single event can be observed. This event was in time coincidence with high energy $\gamma$ 's detected by other bolometers in the same setup, and, therefore, it was likely produced by a muon interaction.

In order to further improve the background suppression, a feasibility study of a muon veto for LUCIFER is ongoing. 


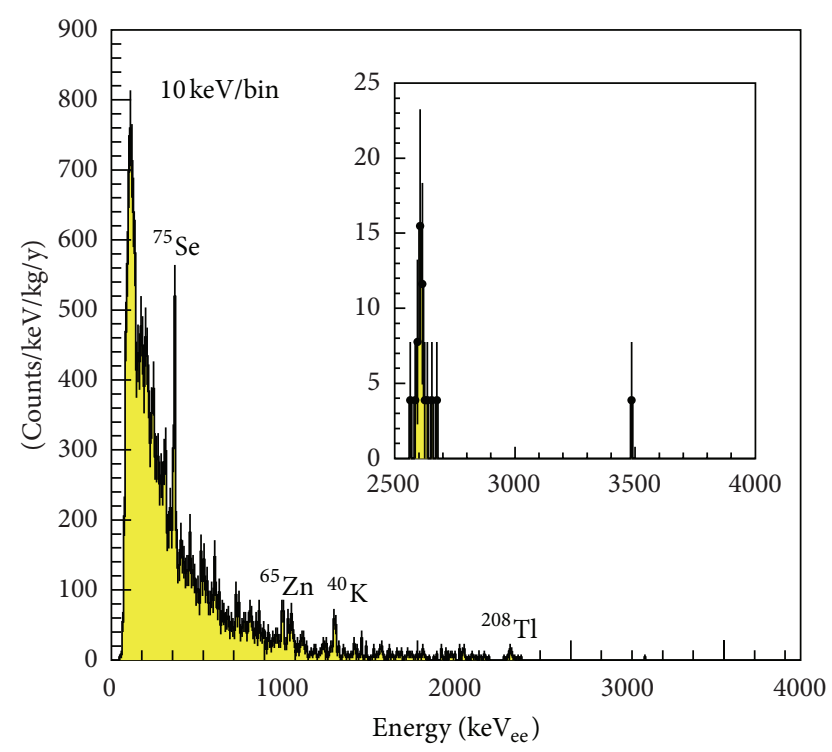

FIGURE 5: $524 \mathrm{~h}$ background run performed with a $431 \mathrm{~g}$ ZnSe crystal. Energy spectrum of $\beta / \gamma$ events. Data are calibrated using $\gamma$ sources. In the inset the magnification of the high energy spectrum.

6.3. $\mathrm{Zn}^{100} \mathrm{MoO}_{4}$. A possible alternative to $\mathrm{ZnSe}$ is $\mathrm{ZnMoO}_{4}$, which is an interesting candidate for the search of $0 v$-DBD of ${ }^{100} \mathrm{Mo}\left(Q_{\beta \beta}=3034 \mathrm{keV}\right)$. This compound, synthesized in the last years, gained a lot of attention because of the excellent bolometric performances and discrimination capabilities.

In this paper, we summarize the results obtained by measuring a $330 \mathrm{~g} \mathrm{ZnMoO}_{4}$ crystal [60], grown by the Nikolaev Institute of Inorganic Chemistry (NIIC, Novosibirsk, Russia), starting with high purity $\mathrm{ZnO}$ (produced by Umicore) and $\mathrm{MoO}_{3}$, synthesized by NIIC.

The FWHM energy resolution achieved with this bolometer ranged from $2.9 \pm 0.4 \mathrm{keV}$ at $583 \mathrm{keV}$ to $6.3 \pm 0.5 \mathrm{keV}$ at $2615 \mathrm{keV}$. Similar results were achieved by other large mass $\mathrm{ZnMoO}_{4}$ crystals, demonstrating the reproducibility of the bolometric performances.

The internal contaminations of the crystals were investigated in a 524-hour background run, showing that $\mathrm{ZnMoO}_{4}$ can reach excellent radiopurity levels even without dedicating too much effort to the radiopurity controls. Only internal contaminations in ${ }^{226} \mathrm{Ra}(27 \pm 6 \mu \mathrm{Bq} / \mathrm{kg})$ and ${ }^{210} \mathrm{~Pb}(700 \pm$ $30 \mu \mathrm{Bq} / \mathrm{kg}$ ) were found.

For the isotopes of ${ }^{232} \mathrm{Th},{ }^{228} \mathrm{Th},{ }^{238} \mathrm{U},{ }^{234} \mathrm{U}$, and ${ }^{230} \mathrm{Th}$, upper limits of $<8 \mu \mathrm{Bq} / \mathrm{kg},<6 \mu \mathrm{Bq} / \mathrm{kg},<6 \mu \mathrm{Bq} / \mathrm{kg},<11 \mu \mathrm{Bq} /$ $\mathrm{kg}$, and $<6 \mu \mathrm{Bq} / \mathrm{kg}$, respectively, were set.

The internal contaminations measured in other prototypes (even if with low exposure) were found to be compatible with the previous results $[61,62]$, showing that the radiopurity levels achievable with $\mathrm{ZnMoO}_{4}$ crystals match the requirements for a low background $0 \nu$-DBD detector.

Finally, the scintillation properties of the bolometer were investigated in several calibration runs performed with a ${ }^{228} \mathrm{Th} \gamma$-source, a smeared $\alpha$ source (similar to the ones described in the previous section), and an Am-Be neutron source to provide high energy $\gamma$ 's.
In Figure 6(a), the relative LY is reported as a function of the heat released in the crystal, showing that an excellent particle discrimination can be achieved. In this run, we measured $\mathrm{LY}(\beta / \gamma)=1.54 \pm 0.01 \mathrm{keV} / \mathrm{MeV}$, which is much larger with respect to $\operatorname{LY}(\alpha)=0.257 \pm 0.002 \mathrm{keV} / \mathrm{MeV}$ (evaluated on the internal $\alpha$ line of ${ }^{210} \mathrm{Po}$ ). The QF for $\alpha$ particles obtained with this crystal is $\mathrm{QF}\left({ }^{210} \mathrm{Po}\right)=0.167 \pm 0.002$, in agreement with the values measured in smaller samples [61].

An interesting feature of $\mathrm{ZnMoO}_{4}$ can be observed in Figure 6(b), where the shape of the heat pulses is reported as a function of the energy deposited in the crystal. This plot shows that the $\alpha$ background rejection provided by the pulse shape discrimination on the heat channel is very similar to the one given by the LY (Figure 6(a)). This feature is induced by the "long" decay constant of the scintillation signal [63] and has several advantages in view of a large mass experiment. Besides the reduction of costs and complexity of the setup, discarding the LDs will also reduce the mass of inert materials next to the detectors, thus possibly reducing the background.

\section{Isotopic Enrichment and Crystal Growth}

The first choice material for LUCIFER is ZnSe crystals grown from ${ }^{82} \mathrm{Se}$ enriched raw material. ZnSe crystals are well known for their extended infrared (IR) transmission and for their scintillating properties and are currently produced for the use in IR optics, optoelectronic devices, and security control systems [64]. ZnSe crystals' growth, however, is quite difficult, and the constraints imposed on their use in DBD experiments add further complications to the obtainment of relatively large crystal samples. Preliminary cryogenic tests show that only $\mathrm{ZnSe}$ crystals grown from the melt can have the crystal perfection needed for good bolometric performances. The growth of $\mathrm{ZnSe}$ crystals from the melt is hampered by several factors.

(i) The $1525^{\circ} \mathrm{C}$ melting point is relatively high and the high total vapor pressure of $\mathrm{ZnSe}\left(2 \mathrm{Bar}\right.$ at $\left.1525^{\circ} \mathrm{C}\right)$ causes containment problems due to the high volatility of components ( $\mathrm{Zn}$ and $\mathrm{Se}$ ) resulting in vaporization and dissociation according to the following reaction:

$$
2(\mathrm{ZnSe})_{\text {solid }} \longleftrightarrow(2 \mathrm{Zn})_{\text {gas }}+\left(\mathrm{Se}_{2}\right)_{\text {gas }} \text {, }
$$

where the different vapor pressures of zinc and selenium lead to deviation from the stoichiometric compositions of the melt during melt growth. The growth from off-stoichiometric melt in nonreactive high pressure enclosures is applied in order to circumvent this problem.

(ii) Melt grown crystals are prone to constitutional supercooling, resulting in local deviations from stoichiometry and formation of inclusions of the excess component. This effect is mainly due to the very sharp homogeneity range in the ZnSe phase diagram [65].

(iii) The low thermal conductivity of $\mathrm{ZnSe}\left(15 \mathrm{~W} / \mathrm{m} \cdot{ }^{\circ} \mathrm{C}\right)$ leads to a very difficult control of the growth interface and therefore to the formation of stresses in 


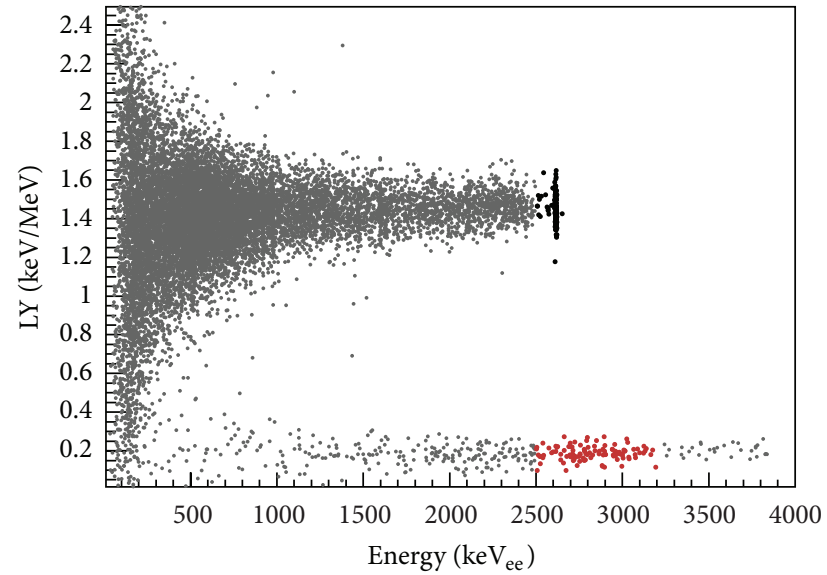

(a)

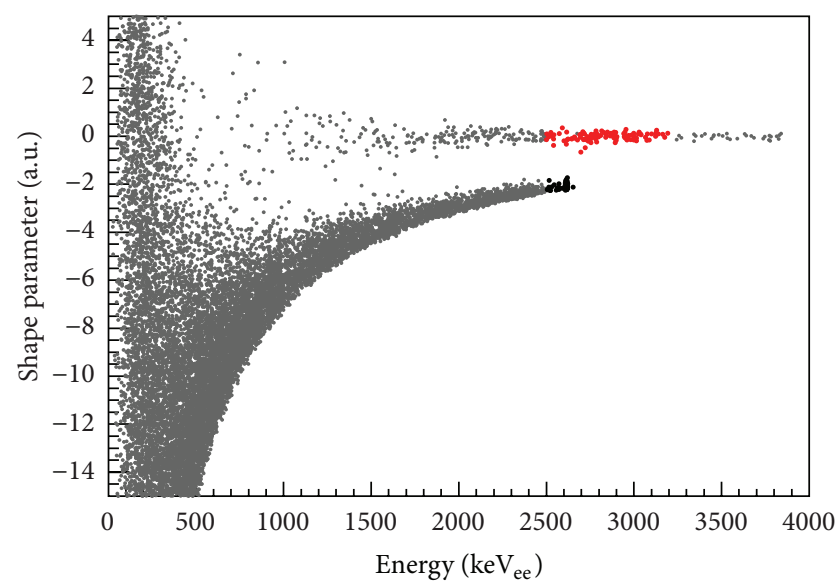

(b)

Figure 6: Particle discrimination in $\mathrm{ZnMoO}_{4}$ obtained in a calibration run with a ${ }^{228} \mathrm{Th} \gamma$-source and a smeared $\alpha$ source. In (a), the LY is reported as a function of the $\gamma$ calibrated heat energy. In (b), the shape of the heat pulses is plotted as a function of the energy, using the same events reported in (a).

the crystal. This is further worsened by a relatively low stacking fault energy $\left(10 \mathrm{erg} / \mathrm{cm}^{2}\right)$, leading to the easy formation of stacking faults (change in the stacking sequence over few interplanar distances) and twinning (stacking faults over several atomic spacings).

(iv) The solid-solid phase transition from wurtzite (hexagonal symmetry) to sphalerite (cubic symmetry) occurring in the proximity of $1400^{\circ} \mathrm{C}[65,66]$ is another cause of extended defects (high dislocation density) in melt grown $\mathrm{ZnSe}$ crystals. The application of low cooling rates in the proximity of the phase transition reduces the formation of defects. The postgrowth annealing further contributes to the recovery of the crystal perfection.

The challenging obtainment of ZnSe crystal for DBD application is further hampered by the necessity to make a dedicated synthesis of the ZnSe compound starting from elemental $\mathrm{Zn}$ and Se $\left({ }^{82} \mathrm{Se}\right.$ enriched). The use of ${ }^{82}$ Se enriched material is required by the relatively low isotopic abundance $(8.73 \%)$ in natural selenium. The enrichment, starting from natural selenium, is ongoing at URENCO, Stable Isotope Group in Almelo, The Netherlands. The starting material is natural $\mathrm{SeF}_{6}$ produced by an external supplier. The technique is based on centrifuges, in a cascade recently updated and fully separated from ${ }^{235} \mathrm{U}$ production in order to avoid any $\mathrm{U}$ and/or Th contamination of the final product. In addition, the centrifuges were flushed before use with fluoride, thus ensuring very good cleaning of the entire cascade. The enriched ${ }^{82} \mathrm{SeF}_{6}$ is further chemically processed to obtain elemental selenium [67]. Care and control are applied at every step of the process from the procurement of natural $\mathrm{SeF}_{6}$ to the delivery of enriched Se in order to guarantee the chemical and radiochemical purity of the final product [68]. The chemical conversion of enriched ${ }^{82} \mathrm{SeF}_{6}$ is the most challenging step in the process of enriched Se production. The selected chemical reaction and corresponding equipment had to be compliant with very strict rules concerning radiocontamination prevention. In addition, the process has to be conducted in such a way to reduce the contamination by impurities defined as critical for the scintillation performance of $\mathrm{ZnSe}$ crystals (Fe, Cr, V, Ni, As, Cu, Mo, Si, and S). The chosen technology required the construction of a dedicated area at the URENCO site in Almelo where the dedicated ${ }^{82} \mathrm{SeF}_{6}$ to ${ }^{82} \mathrm{Se}$ conversion rig will be operated.

Presently, the overall chemical purity turns out to be better than $99.8 \%$ on trace metal base; in particular, the concentrations of ${ }^{238} \mathrm{U}$ and ${ }^{232} \mathrm{Th}$ fall below $10^{-10} \mathrm{~g} / \mathrm{g}$ and the critical impurities have concentrations below the accepted limits for a good scintillation performance of $\mathrm{ZnSe}$ crystals [68]. The distribution of different phases of the very complex process of ZnSe crystal production is currently fixed, and the related production contracts are in progress or under discussion. The diagram of the production of enriched crystals is given in Figure 7.

The crystals discussed in this work were grown from the melt, in graphite crucibles using the Bridgman technique in a vertical furnace under inert gas (Argon) pressured up to $15 \mathrm{Bar}$ in a temperature gradient with the maximum temperature reaching $1575^{\circ} \mathrm{C}$ in the melt zone. The growth rate (variable along crucible length) was $2-5 \mathrm{~mm}$ /hour. After growth, crystals were annealed in the same furnace and then cut and polished to the standard (LUCIFER) dimensions: $45 \mathrm{~mm}$ diameter and $55 \mathrm{~mm}$ length for a total volume of $87.4 \mathrm{~cm}^{3}$ and a mass of $460.7 \mathrm{~g}$ in the case of natural $\mathrm{ZnSe}$. The expected mass of $\mathrm{ZnSe}$ crystals grown from enriched material (enriched selenium with $95 \%{ }^{82} \mathrm{Se}$ content) with standard dimensions is $469.98 \mathrm{~g}$ corresponding to $248.1 \mathrm{~g}$ content of ${ }^{82} \mathrm{Se}$. Table 1 gives the mass balance of $\mathrm{ZnSe}$ crystals grown from natural and enriched raw material.

The amount of enriched Se foreseen for the experiment is $15 \mathrm{~kg}$. For an estimated yield of ZnSe synthesis of $97 \%$ (3\% irrecoverable loss of enriched Se, i.e., $14.55 \mathrm{~kg}$ effective enriched Se embodied in $26.2 \mathrm{~kg}$ of enriched $\mathrm{ZnSe}$ powder) and an estimated yield of crystal growth and processing of 


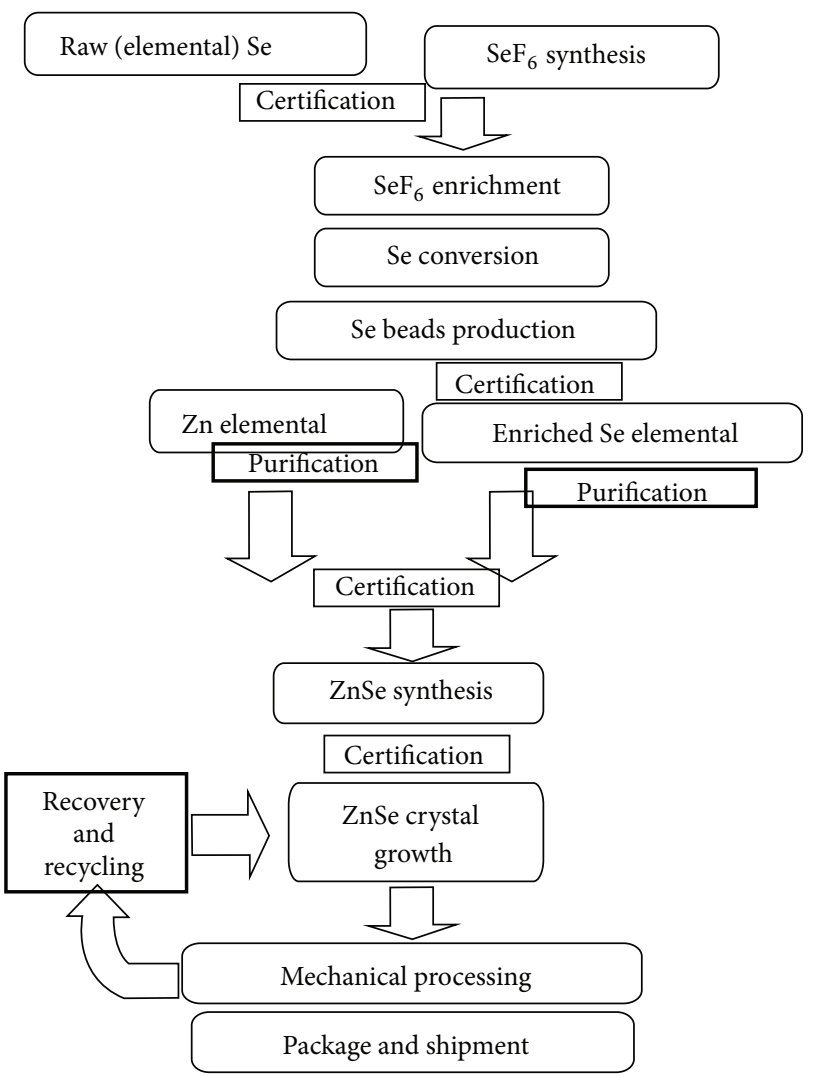

FIGURE 7: Production diagram of enriched ZnSe crystals.

$65 \%$ (35\% irrecoverable loss of enriched $\mathrm{ZnSe}$ ), it comes out that the total expected enriched crystal mass is $16.9 \mathrm{~kg}(8.9 \mathrm{~kg}$ content of ${ }^{82} \mathrm{Se}$ ) embodied in a total number of 36 crystals with standard dimensions. The relatively low yield of crystal production comes from the irrecoverable loss in evaporated material during the growth process and the losses during the mechanical processing (cutting, shaping, and polishing) of the as grown crystal to the final crystal sample.

\section{Thermistor Production}

Neutron transmutation doped (NTD) thermistors are semiconductor devices doped close to the metal to insulator transition. Melt-doped Ge crystals cannot achieve the necessary uniformity due to a variety of dopant segregation effects. The only technique available for producing a uniform doping is to produce the dopant by bombarding the Ge crystal with thermal neutrons. The most important aspect of this process is that ${ }^{70} \mathrm{Ge}$ transmutes into ${ }^{71} \mathrm{Ga}$, an acceptor, and ${ }^{74} \mathrm{Ge}$ transmutes into ${ }^{75} \mathrm{As}$, a donor, the primary active dopants in NTD Ge. The optimization of the detector performance is strictly related to the characteristics of the NTDs.

The thermal signal from $\mathrm{ZnSe}$ crystals and light detectors will be read out using NTDs that must be optimized for such specific application. The variation of resistivity of heavily doped thermistors as function of temperature, $T$, is well represented by the formula

$$
\rho(T)=\rho_{0} \cdot e^{\left(T_{0} / T\right)^{\gamma}}
$$

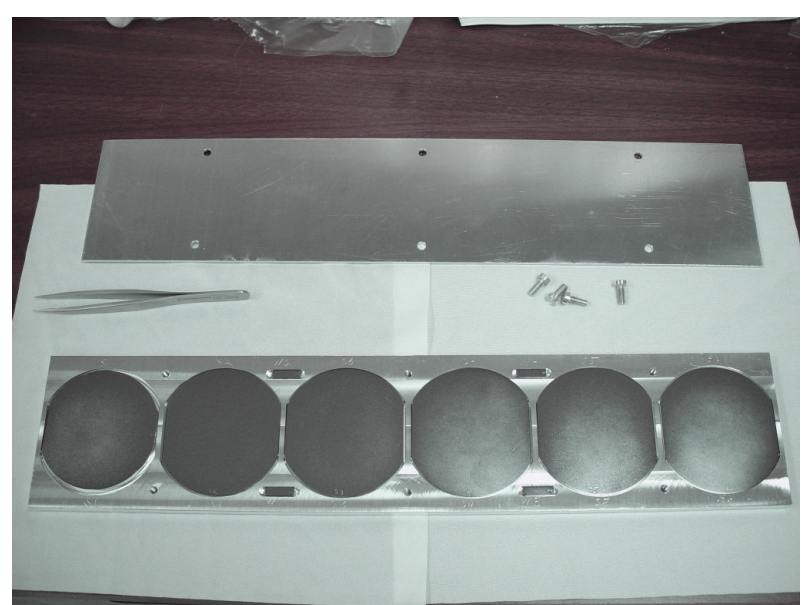

FIGURE 8: Six out of nine wafers in the Al holder before the neutron irradiation that took place at MIT between 2012 and 2013.

where $\gamma$ is a parameter that normally shows a value around $1 / 2$ and $\rho_{0}$ and $T_{0}$ are directly correlated with the doping profile of the NTD. In particular, the more critical parameter is $T_{0}$, due to its extremely strong dependence on the total neutron fluence.

Starting from our previous experience [21], there are precise indications for the characteristics of sensors that will give us the best performance in our experimental temperature range. In fact, we have chosen a $T_{0}$ of the order of $3.8 \mathrm{~K}$ that, together with the fixed dimensions of the thermistors $(3 \times 3 \times$ $1 \mathrm{~mm}^{3}$ ), gives a working resistance of few $\mathrm{M} \Omega$ at $15 \mathrm{mK}$.

In order to produce thermistors with the adequate features, we proceeded in two independent ways.

8.1. New Production. A completely new production has begun in 2012, starting from nine HPGE Ge wafers. The shape of the single wafer is close to a circular shape with $\oslash=66 \mathrm{~mm}$ and $3.2 \mathrm{~mm}$ thickness (see Figure 8 ).

All the neutron irradiations (ten independent runs) were performed at MIT Nuclear Reactor Laboratory between February 2012 and March 2013. Considering that a change in the total dose of neutrons of only $2.5 \%$ will change the value of $T_{0}$ by 1 unity (our $T_{0}$ acceptance window is between 3.3 and $4.3 \mathrm{~K}$ ) and that the whole process of production (irradiation + cooling time) takes 2 years, we asked for four different doses: 3 wafers at $-7 \%$ of the nominal dose, 2 wafers at $-3 \%$ of the nominal does, 3 wafers at the nominal dose, and 1 wafer at the $+2 \%$ of the nominal dose. From the single wafer, more than 500 thermistors can be obtained. The first cryogenic characterizations of the irradiated wafers will take place in October 2013.

8.2. NTD Fine Tuning. We have a previous "old" NTD wafer that is slightly underdoped, with $T_{0}=4.6 \mathrm{~K}$. This value, which is considered slightly too high for LUCIFER, can be corrected by reirradiating the sample with a total fluence of $\sim 0.9 \cdot 10^{17} \mathrm{n} / \mathrm{cm}^{2}$. This is not a large value but the new irradiation phase needs a very precise integral flux control. Due to the fact that MIT NRL cannot provide this fine 
TABLE 1: Mass balance of ZnSe crystals grown from natural and enriched raw material. The baseline choice for LUCIFER is highlighted in bold.

\begin{tabular}{|c|c|c|c|c|c|c|c|c|c|c|}
\hline \multirow[b]{2}{*}{ Dia. $(\mathrm{cm})$} & \multirow[b]{2}{*}{ Height $(\mathrm{cm})$} & \multirow[b]{2}{*}{ Vol. $\left(\mathrm{cm}^{3}\right)$} & \multicolumn{4}{|c|}{ Natural } & \multicolumn{4}{|c|}{ Enriched 95\% } \\
\hline & & & Mass (g) & $m(\mathrm{Se})(\mathrm{g})$ & $m\left({ }^{82} \mathrm{Se}\right)(\mathrm{g})$ & $N\left({ }^{82} \mathrm{Se}\right)\left(10^{23}\right)$ & Mass (g) & $m(\mathrm{Se})(\mathrm{g})$ & $m\left({ }^{82} \mathrm{Se}\right)(\mathrm{g})$ & $N\left({ }^{82} \mathrm{Se}\right)\left(10^{-23}\right)$ \\
\hline 4.5 & 5 & 79.5 & 418.8 & 229.1 & 16.5 & 1.22 & 427.2 & 237.5 & 225.6 & 16.6 \\
\hline 4.5 & 5.5 & 87.4 & 460.7 & 252.0 & 18.2 & 1.34 & 469.9 & 261.2 & 248.1 & 18.2 \\
\hline 4.5 & 6 & 95.4 & 502.6 & 274.9 & 19.9 & 1.46 & 512.7 & 284.9 & 270.7 & 19.9 \\
\hline
\end{tabular}

tuning, we found an alternative nuclear reactor. To fulfill the previously mentioned requests, we characterized a selected neutron irradiation channel in the TRIGA Mark II nuclear reactor of the LENA (Laboratorio Energia Nucleare Applicata) Laboratory of Pavia University. After a long characterization of the neutron spectrum of the various irradiation channels [69] (in terms of flux and energy spectrum), the irradiation of the "old" NTD wafer was started in July 2013. Due to the cooling time, we will make the first test of those thermistors by the beginning of 2014 .

\section{Background and Sensitivity}

The sensitivity of a given $0 v$-DBD experiment is defined as the half-life corresponding to the signal that could be emulated by a background fluctuation of a chosen significance level, expressed in numbers of the Gaussian standard deviations $\left(n_{\sigma}\right)$

$$
T_{1 / 2}^{0 v}=\frac{\ln 2}{n_{\sigma}} \frac{N_{A} a \eta \epsilon}{W} \sqrt{\frac{M T}{B \Delta E}} f(\delta E),
$$

where $\eta$ is the stoichiometric coefficient of the DBD candidate, $a$ is the DBD candidate isotopic abundance, $N_{A}$ is Avogadro's number, $W$ is the molecular weight of the active mass, $B$ is the background rate per unit mass and energy, $M$ is the detector mass, $T$ is the live time, $\Delta E$ is the FWHM energy resolution, $\epsilon$ is the detection efficiency, and $f(\delta E)$ is the fraction of signal events that fall in an energy window $\delta E$ around the $Q$-value.

The sensitivity $T_{1 / 2}^{0 v}$ from (3) is then translated into an effective Majorana mass sensitivity

$$
\left\langle m_{\nu}\right\rangle=\frac{m_{e}}{\left(T_{1 / 2}^{0 \nu} F_{N}^{0 \nu}\right)^{1 / 2}},
$$

where $m_{e}$ is the electron mass and $F^{0 v}$ is the nuclear factor of merit, defined as $F_{N}^{0 v}=G^{0 \nu}\left|M^{0 \nu}\right|^{2}$. The quantities $G^{0 v}$ and $M^{0 v}$ represent, respectively, the two-body phase-space factor and the $0 v$-DBD nuclear matrix element (NME). While $G^{0 v}$ can be calculated with reasonable accuracy, the NME value is strongly dependent on the nuclear model used for its evaluation.

Equation (3) holds if the number of background counts is large enough so that its distribution can be considered to be Gaussian. In the limit of zero background counts in the region of interest (see e.g., [70]), the above formula reduces to

$$
T_{1 / 2}^{0 v}=-\frac{\ln 2}{\ln (1-\text { C.L. } / 100)} \frac{N_{A} a \eta \epsilon}{W} \operatorname{MTf}(\delta E) .
$$

TABLE 2: LUCIFER experimental parameters for the two baseline $0 v$ DBD candidates. For each isotope, we quote the type of scintillating crystal, the total detector mass, the molecular weight of the active mass $(W)$, the isotopic abundance assuming enrichment $(a)$, the efficiency, and the energy resolution.

\begin{tabular}{lcccccc}
\hline Isotope & Crystal & $\begin{array}{c}\text { Mass } \\
(\mathrm{kg})\end{array}$ & $W$ & $a(\%)$ & $\epsilon(\%)$ & $\begin{array}{c}\text { FWHM } \\
(\mathrm{keV})\end{array}$ \\
\hline${ }^{82} \mathrm{Se}$ & $\mathrm{ZnSe}$ & 17 & 144.34 & 95 & 76 & 10 \\
${ }^{100} \mathrm{Mo}$ & $\mathrm{ZnMoO}_{4}$ & 14 & 225.32 & 95 & 77 & 5 \\
\hline
\end{tabular}

To calculate the sensitivity of LUCIFER to $0 v$-DBD, we need to define the assumptions on the experimental parameters and quantities of (3), as well as the setup of the experiment. These assumptions are summarized in Table 2 . The value of $f(\delta E)$ for $\delta E=\Delta E$ is 0.76 . On the basis of what was discussed in Section 3, we assume a tower-like detector of 36 crystals $\left(\mathrm{ZnSe}\right.$ or $\mathrm{ZnMoO}_{4}$ ) arranged in a modular configuration of 9 floors with 4 crystals each, held together by a copper structure. Light detectors, consisting of Ge ultrapure wafers of about $180 \mu \mathrm{m}$ thickness and $44 \mathrm{~mm}$ diameter, will be faced to each of the scintillating crystals, which are wrapped in a reflecting sheet to improve the light collection efficiency.

The detection efficiency $(\epsilon)$ of $\mathrm{ZnSe}$ and $\mathrm{ZnMoO}_{4}$ crystals was estimated by means of GEANT4 Monte Carlo simulations and represents the fraction of $0 v$-DBD decays, where the total energy of the two emitted electrons is contained within the crystal. The energy resolution (FWHM) is assumed to be $10 \mathrm{keV}$ for $\mathrm{ZnSe}$ crystals and $5 \mathrm{keV}$ for $\mathrm{ZnMoO}_{4}$ crystals.

The LUCIFER tower will be inserted into a dilution refrigerator made of few nested cylindrical copper thermal shields. A $10 \mathrm{~cm}$ thick lead disk, placed just above the tower, provides supplementary shielding against the radioactivity of the various components of the refrigerator located above the detectors: the dilution unit, the pumping lines, and the cabling system. Outside the refrigerator, a $20 \mathrm{~cm}$ (minimum) thick lead shield and a $10 \mathrm{~cm}$ thick borated polyethylene shield will be used to absorb $\gamma$ 's and neutrons from the environmental background of the LNGS experimental hall.

Generally, the background components for an underground DBD experiment can be divided into the so-called near sources (radioactive contaminations of crystals and copper mounting structures), far sources (radioactive contaminations of refrigerator and its external shields), and environmental sources (muons, neutrons, and gamma rays fluxes at the experimental site).

GEANT4 simulations of the various background components for a detector setup similar to what was described here 
TABLE 3: LUCIFER half-life background-fluctuation sensitivity in 5 or $10 \mathrm{yr}$ at $90 \%$ C.L., calculated using (5), and the corresponding ranges of the Majorana neutrino mass, calculated considering the most recent NME [71-77] calculations.

\begin{tabular}{lccc}
\hline Crystal & Live time (y) & $\begin{array}{c}\text { Half-life sensitivity } \\
\left(10^{26} \mathrm{y}\right)\end{array}$ & $\left\langle m_{\nu}\right\rangle(\mathrm{meV})$ \\
\hline $\mathrm{ZnSe}$ & 5 & 0.6 & $65-194$ \\
& 10 & 1.2 & $46-138$ \\
$\mathrm{ZnMoO}_{4}$ & 5 & 0.3 & $60-170$ \\
& 10 & 0.6 & $42-120$ \\
\hline
\end{tabular}

have shown [61] that, exploring the background discrimination potential of the scintillating bolometers discussed in this work and given the radiopurity of the selected crystals and the detector's materials presently available, a background index of $10^{-4}$ counts $/ \mathrm{kg} / \mathrm{keV} / \mathrm{y}$ in the ROI is within reach.

However, as pointed out in Section 3, the detector will be mounted in the Cuoricino cryostat. This cryostat, unfortunately, was constructed in the late 1980s, and at that time, the materials' selection requirements were not so stringent as nowadays. This implies that, even with changing the most internal $\mathrm{Cu}$ thermal and vacuum shields of the cryostat, the ultimate background will be dominated by the liquid helium Dewar in which the dilution unit is placed. The unavoidable background induced by ${ }^{214} \mathrm{Bi} \gamma$ 's generated in the Dewar is evaluated to be $\leq 1.5 \times 10^{-3}$ counts $/ \mathrm{kg} / \mathrm{keV} / \mathrm{y}$.

For $\mathrm{ZnMoO}_{4}$ crystals, the dominant background contribution comes from an additional source with respect to the ones mentioned above: the accidental pileup of $2 v$-DBD decays [62], given the slow time response of bolometric detectors and the relatively "fast" $2 v$-DBD decay of ${ }^{100} \mathrm{Mo}$.

It has been shown, however, that a faster time response of light detectors can improve the pileup discrimination potential and reduce the background induced by ${ }^{100} \mathrm{Mo} 2 \nu$ DBD to well below $10^{-4}$ counts $/ \mathrm{kg} / \mathrm{keV} / \mathrm{y}$ [71].

From the numbers in Table 2 and assuming a background index in the ROI of $10^{-3}$ counts $/ \mathrm{kg} / \mathrm{keV} / \mathrm{y}$, we can estimate the number of background counts in the ROI to be $\sim 1(\sim 2)$ for a live time of 5 (10) yr. This means we cannot use the Gaussian approximation for the background fluctuations, but indeed we need to use the zero-background approximation.

In Table 3, we report the LUCIFER half-life backgroundfluctuation sensitivity at $90 \%$ C.L. in 5 or $10 \mathrm{yr}$, calculated using (5). From the value of $T_{1 / 2}^{0 \nu}$, we extracted the corresponding limit on the effective Majorana neutrino mass that could be set by LUCIFER, considering the spread on most recent NME calculations [72-78] and the phase space factor from [79].

Moreover, LUCIFER can be considered a demonstrator of the scintillating bolometer technology with a significant mass and a full test of all the critical experimental issues of this technique and could lead the way towards a new generation of $0 v$-DBD experiments with scintillating bolometers able to scrutinize the inverted hierarchy region of the Majorana neutrino mass spectrum.

\section{Conclusions}

In this paper, we have exposed a set of relevant results which show how the LUCIFER technology is now mature not only for the fabrication of a LUCIFER demonstrator at the $10 \div$ $15 \mathrm{~kg}$ scale (immediate objective of the LUCIFER program) but also for a much larger next-generation experiment.

The activity described here has allowed us to set the bases for the procurement of a considerable amount of ${ }^{82} \mathrm{Se}$, including for the first time a European company in the extremely exclusive group of potential producers of enriched isotopes for $0 v$-DBD search. We have studied in detail the production chain which allows us to embed the enriched isotope into the final ZnSe crystals, basic elements of the $0 v$-DBD detectors. The technical performances of the $\mathrm{ZnSe}$ scintillating bolometers, in terms of energy resolution and alpha-particle rejection factor, are fully compatible with an extremely sensitive $0 v$-DBD experiment. The present indications on the background are also very promising and show that a background index of at least $10^{-3}$ counts $/ \mathrm{kg} / \mathrm{keV} / \mathrm{y}$ is reachable.

We have presented also results on an alternative isotope and compound, that is, ${ }^{100}$ Mo embedded in $\mathrm{ZnMoO}_{4}$ crystals, which competes with the ${ }^{82} \mathrm{Se}$ way in view of a next-generation experiment, offering better energy resolution thanks to superior crystal features. The radiopurity of the crystals studied so far is also quite promising.

Coming to more technical aspects, we have fully determined the structure of the light detectors and fabricated successful prototypes of these devices. We have also started the production of a considerable amount of neutron transmutation doped thermistors (the temperature sensors used both in the heat and in the light channels), largely sufficient to equip all the scintillating crystals and the light detectors foreseen in the LUCIFER demonstrator.

A detailed study of the background and of the sensitivity, assuming detector configuration and performance based on the features-reported here-exhibited by working prototypes, shows that the LUCIFER demonstrator can approach the inverted hierarchy region both in the ZnSe and in the $\mathrm{ZnMoO}_{4}$ versions. In the former case, the required amount of isotope is available. A well-founded extrapolation to the sensitivities of larger setups, limited essentially by the cost of the enrichment, indicates clearly that arrays of $\mathrm{ZnSe}$ and/or $\mathrm{ZnMoO}_{4}$ scintillating bolometers are viable candidates for a next-generation $0 v$-DBD experiment, capable of fully exploring the inverted hierarchy region of the neutrino mass pattern.

\section{Acknowledgments}

This work was made in the frame of the LUCIFER experiment, funded by the European Research Council under the European Union's Seventh Framework Programme (FP7/ 2007-2013)/ERC Grant Agreement no. 247115. Thanks are due to E. Tatananni, A. Rotilio, A. Corsi, B. Romualdi, and $\mathrm{M}$. Guetti for continuous and constructive help in the overall setup construction. 


\section{References}

[1] M. Goeppert-Mayer, "Double beta-disintegration," Physical Review, vol. 48, no. 6, pp. 512-516, 1935.

[2] A. S. Barabash, "Precise half-life values for two-neutrino double- $\beta$ decay," Physical Review C, vol. 81, no. 3, Article ID 035501, 2010.

[3] F. T. Avignone III, S. R. Elliott, and J. Engel, "Double beta decay, Majorana neutrinos, and neutrino mass," Reviews of Modern Physics, vol. 80, no. 2, pp. 481-516, 2008.

[4] J. J. Gómez-Cadenas, J. Martín-Albo, M. Mezzetto, F. Monrabal, and M. Sorel, "The search for neutrinoless double beta decay," Rivista del Nuovo Cimento, vol. 35, no. 2, pp. 29-98, 2012.

[5] A. Giuliani and A. Poves, "Neutrinoless double-beta decay," Advances in Hight Energy Physics, vol. 2012, Article ID 857016, 38 pages, 2012.

[6] J. D. Vergados, H. Ejiri, and F. Simkovic, "Theory of neutrinoless double-beta decay," Reports on Progress in Physics, vol. 75, Article ID 106301, 2012.

[7] W. H. Furry, "On transition probabilities in double betadisintegration," Physical Review, vol. 56, no. 12, pp. 1184-1193, 1939.

[8] E. Majorana, "Teoria simmetrica dell'elettrone e del positrone," Il Nuovo Cimento, vol. 14, no. 4, pp. 171-184, 1937.

[9] J. Schechter and J. W. F. Valle, "Neutrinoless double- decay in $\mathrm{SU}(2) \times \mathrm{U}(1)$ theories," Physical Review D, vol. 25, no. 11, pp. 2951-2954, 1982.

[10] M. Fukugita and T. Yanagida, "Barygenesis without grand unification," Physics Letters B, vol. 174, no. 1, pp. 45-47, 1986.

[11] H. V. Klapdor-Kleingrothaus and I. V. Krivosheina, "The evidence for the observation of $0 \nu \beta \beta$ decay: the identification of $0 \nu \beta \beta$ events from the full spectra," Modern Physics Letters A, vol. 21, no. 20, pp. 1547-1566, 2006.

[12] A. Gando, Y. Gando, H. Hanakago et al., "Limit on neutrinoless $\beta \beta$ decay of ${ }^{136} \mathrm{Xe}$ from the first phase of KamLAND-Zen and comparison with the positive claim in ${ }^{76} \mathrm{Ge}$," Physical Review Letters, vol. 110, no. 6, Article ID 062502, 2013.

[13] M. Agostini, M. Allardt, E. Andreotti et al., "Results on neutrinoless double beta decay of ${ }^{76} \mathrm{Ge}$ from GERDA Phase I," Physical Review Letters, vol. 111, Article ID 122503, 2013.

[14] R. Arnold, C. Augier, J. Baker et al., "Probing new physics models of neutrinoless double beta decay with SuperNEMO," European Physical Journal C, vol. 70, no. 4, pp. 927-943, 2010.

[15] C. Arnaboldi, F. T. Avignone III, J. Beeman et al., "CUORE: a cryogenic underground observatory for rare events," Nuclear Instruments and Methods in Physics Research A, vol. 518, no. 3, pp. 775-798, 2004.

[16] M. Auger, D. J. Auty, P. S. Barbeau et al., "Search for neutrinoless double-beta decay in ${ }^{136}$ Xe with EXO-200," Physical Review Letters, vol. 109, no. 3, Article ID 032505, 2012.

[17] V. Álvarez, F. I. G. M. Borges, S. Cárcel et al., "Initial results of NEXT-DEMO, a large-scale prototype of the NEXT-100 experiment," Journal of Instrumentation, vol. 8, Article ID P04002, 2013.

[18] C. Kraus and S. J. M. Peeters, “The rich neutrino programme of the SNO+ experiment," Progress in Particle and Nuclear Physics, vol. 64, no. 2, pp. 273-277, 2010.

[19] C. Enss and D. McCammon, "Physical principles of low temperature detectors: ultimate performance limits and current detector capabilities," Journal of Low Temperature Physics, vol. 151, no. 1-2, pp. 5-24, 2008.
[20] L. Cardani, L. Gironi, J. W. Beeman et al., "Performance of a large $\mathrm{TeO}_{2}$ crystal as a cryogenic bolometer in searching for neutrinoless double beta decay," Journal of Instrumentation, vol. 7, no. 1, Article ID P01020, 2012.

[21] E. Andreotti, C. Arnaboldi, F. T. Avignone III et al., " ${ }^{130}$ Te neutrinoless double-beta decay with CUORICINO," Astroparticle Physics, vol. 34, no. 11, pp. 822-831, 2011.

[22] M. Clemenza, C. Maiano, L. Pattavina, and E. Previtali, "Radoninduced surface contaminations in low background experiments," The European Physical Journal C, vol. 71, p. 1805, 2011.

[23] C. Bucci, S. Capelli, M. Carrettoni et al., "Background study and Monte Carlo simulations for large-mass bolometers," European Physical Journal A, vol. 41, no. 2, pp. 155-168, 2009.

[24] G. Angloher, M. Bauer, I. Bavykina et al., "Results from $730 \mathrm{~kg}$ days of the CRESST-II Dark Matter search," European Physical Journal C, vol. 72, no. 4, article 1971, 2012.

[25] J. Amaré, B. Beltrán, J. M. Carmona et al., "Recent developments on scintillating bolometers for WIMP searches: ROSEBUD status," Journal of Physics: Conference Series, vol. 39, no. 1, pp. 133-135, 2006.

[26] S. Pirro, J. W. Beeman, S. Capelli, M. Pavan, E. Previtali, and P. Gorla, "Scintillating double-beta-decay bolometers," Physics of Atomic Nuclei, vol. 69, no. 12, pp. 2109-2116, 2006.

[27] C. Arnaboldi, C. Brofferio, O. Cremonesi et al., "A novel technique of particle identification with bolometric detectors," Astroparticle Physics, vol. 34, no. 11, pp. 797-804, 2011.

[28] L. Gironi, C. Arnaboldi, J. W. Beeman et al., "Performance of $\mathrm{ZnMoO}_{4}$ crystal as cryogenic scintillating bolometer to search for double beta decay of molybdenum," Journal of Instrumentation, vol. 5, no. 11, Article ID P11007, 2010.

[29] C. Arnaboldi, J. W. Beeman, O. Cremonesi et al., " $\mathrm{CdWO}_{4}$ scintillating bolometer for double beta decay: light and heat anticorrelation, light yield and quenching factors," Astroparticle Physics, vol. 34, no. 3, pp. 143-150, 2010.

[30] C. Arnaboldi, S. Capelli, O. Cremonesi et al., "Characterization of ZnSe scintillating bolometers for double beta decay," Astroparticle Physics, vol. 34, no. 6, pp. 344-353, 2011.

[31] C. Arnaboldi, G. Pessina, and S. Pirro, "The cold preamplifier set-up of CUORICINO: towards 1000 channels," Nuclear Instruments and Methods in Physics Research A, vol. 559, no. 2, pp. 826-828, 2006.

[32] C. Arnaboldi, C. Bucci, S. Capelli et al., "The front-end readout for CUORICINO, an array of macro-bolometers and MIBETA, an array of $\mu$-bolometers," Nuclear Instruments and Methods in Physics Research A, vol. 520, no. 1-3, pp. 578-580, 2004.

[33] C. Arnaboldi, G. Boella, E. Panzeri, and G. Pessina, "JFET transistors for low-noise applications at low frequency," IEEE Transactions on Nuclear Science, vol. 51, no. 6, pp. 2975-2982, 2004.

[34] C. Arnaboldi, A. Fascilla, M. W. Lund, and G. Pessina, “Temperature characterization of deep and shallow defect centers of low noise silicon JFETs," Nuclear Instruments and Methods in Physics Research A, vol. 517, no. 1-3, pp. 313-336, 2004.

[35] A. Giachero, C. Gotti, M. Maino, and G. Pessina, "Modelling high impedance connecting links and cables below $1 \mathrm{~Hz}$," Journal of Instrumentation, vol. 7, Article ID P08004, 2012.

[36] G. Pessina, "Room temperature differential voltage sensitive preamplifier for large mass bolometric detectors," Nuclear Instruments and Methods in Physics Research A, vol. 444, no. 1, pp. 132-135, 2000. 
[37] A. Alessandrello, C. Brofferio, C. Bucci et al., "A low de drift read-out system for a large mass bolometric detector," IEEE Transactions on Nuclear Science, vol. 44, no. 3, pp. 416-423, 1997.

[38] A. Alessandrello, C. Brofferio, D. V. Camin et al., "Measuring thermistor resistance with very low D.C. power dissipation," Cryogenics, vol. 37, no. 1, pp. 27-31, 1997.

[39] C. Arnaboldi, C. Bucci, O. Cremonesi et al., "Low-frequency noise characterization of very large value resistors," IEEE Transactions on Nuclear Science, vol. 49, no. 4, pp. 1808-1813, 2002.

[40] C. Arnaboldi and G. Pessina, "The design of the input stage for the very front-end of the CUORE experiment," Journal of Low Temperature Physics, vol. 151, no. 3-4, pp. 964-970, 2008.

[41] A. Alessandrello, C. Brofferio, C. Bucci et al., "Programmable front-end system for arrays of bolometers," Nuclear Instruments and Methods in Physics Research A, vol. 444, no. 1, pp. 111-114, 2000.

[42] C. Arnaboldi, C. Bucci, J. W. Campbell et al., "The programmable front-end system for CUORICINO, an array of large-mass bolometers,' IEEE Transactions on Nuclear Science, vol. 49, no. 5, pp. 2440-2447, 2002.

[43] C. Arnaboldi, M. Cariello, S. Di Domizio, A. Giachero, and G. Pessina, "A programmable multichannel antialiasing filter for the CUORE experiment," Nuclear Instruments and Methods in Physics Research A, vol. 617, no. 1-3, pp. 327-328, 2010.

[44] G. Pessina, "Low-noise, low drift, high precision linear bipolar $( \pm 10 \mathrm{~V})$ voltage supply/reference for cryogenic front-end apparatus," Review of Scientific Instruments, vol. 70, no. 8, pp. 34733478, 1999.

[45] A. Alessandrello, V. Bashkirov, C. Brofferio et al., "Development of a thermal scintillating detector for double beta decay of ${ }^{48} \mathrm{Ca}$," Nuclear Physics B, vol. 28, no. 1, pp. 233-235, 1992.

[46] C. Bobin, I. Berkes, J. P. Hadjout, N. Coron, J. Leblanc, and P. De Marcillac, "Alpha/gamma discrimination with a $\mathrm{CaF} 2(\mathrm{Eu})$ target bolometer optically coupled to a composite infrared bolometer," Nuclear Instruments and Methods in Physics Research A, vol. 386, no. 2-3, pp. 453-457, 1997.

[47] S. Pirro, C. Arnaboldi, J. W. Beeman, and G. Pessina, "Development of bolometric light detectors for double beta decay searches," Nuclear Instruments and Methods in Physics Research A, vol. 559, no. 2, pp. 361-363, 2006.

[48] J. W. Beeman, A. Gentils, A. Giuliani et al., "Effect of $\mathrm{SiO}_{2}$ coating in bolometric Ge light detectors for rare event searches," Nuclear Instruments and Methods in Physics Research A, vol. 709, pp. 22-28, 2013.

[49] J. W. Beeman, F. Bellini, N. Casali et al., "Characterization of bolometric light detectors for rare event searches," Journal of Instrumentation, vol. 8, Article ID P07021, 2013.

[50] S. Pirro, A. Alessandrello, C. Brofferio et al., "Vibrational and thermal noise reduction for cryogenic detectors," Nuclear Instruments and Methods in Physics Research A, vol. 444, no. 1, pp. 331-335, 2000.

[51] S. Pirro, "Further developments in mechanical decoupling of large thermal detectors," Nuclear Instruments and Methods in Physics Research A, vol. 559, no. 2, pp. 672-674, 2006.

[52] C. Arnaboldi, C. Bucci, S. Capelli et al., "The temperature stabilization system of CUORICINO: an array of macro bolometers," IEEE Transactions on Nuclear Science, vol. 52, no. 5, pp. 16301637, 2005.

[53] A. Alessandrello, C. Brofferio, C. Bucci et al., "Methods for response stabilization in bolometers for rare decays," Nuclear Instruments and Methods in Physics Research A, vol. 412, no. 2-3, pp. 454-464, 1998.
[54] C. Arnaboldi, G. Pessina, and E. Previtali, "A programmable calibrating pulse generator with multi-outputs and very high stability," IEEE Transactions on Nuclear Science, vol. 50, no. 4, pp. 979-986, 2003.

[55] C. Arnaboldi, A. Giachero, C. Gotti, and G. Pessina, "A very high performance stabilization system for large mass bolometer experiments," Nuclear Instruments and Methods in Physics Research A, vol. 652, pp. 306-309, 2011.

[56] E. Gatti and P. F. Manfredi, "Processing the signals from solidstate detectors in elementary-particle physics," La Rivista del Nuovo Cimento, vol. 9, no. 1, pp. 1-146, 1986.

[57] V. Radeka and N. Karlovac, "Least-square-error amplitude measurement of pulse signals in presence of noise," Nuclear Instruments and Methods, vol. 52, no. 1, pp. 86-92, 1967.

[58] G. Piperno, S. Pirro, and M. Vignati, "Optimizing the energy threshold of light detectors coupled to luminescent bolometers," Journal of Instrumentation, vol. 6, no. 10, Article ID P10005, 2011.

[59] J. W. Beeman, F. Bellini, L. Cardani et al., "Performances of a large mass ZnSe bolometer to search for rare events," Journal of Instrumentation, vol. 8, Article ID P05021, 2013.

[60] J. W. Beeman, F. Bellini, C. Brofferio et al., "Performances of a large mass $\mathrm{ZnMoO}_{4}$ scintillating bolometer for a next generation $0 \nu$ DBD experiment," The European Physical Journal C, vol. 72, p. 2142, 2012.

[61] J. W. Beeman, F. Bellini, S. Capelli et al., " $\mathrm{ZnMoO}_{4}$ : a promising bolometer for neutrinoless double beta decay searches," Astroparticle Physics, vol. 35, no. 12, pp. 813-820, 2012.

[62] J. W. Beeman, F. A. Danevich, V. Y. Degoda et al., "A nextgeneration neutrinoless double beta decay experiment based on $\mathrm{ZnMoO}_{4}$ scintillating bolometers," Physics Letters B, vol. 710, no. 2, pp. 318-323, 2012.

[63] L. Gironi, "Pulse shape analysis with scintillating bolometers," The Journal of Low Temperature Physics, vol. 167, pp. 504-509, 2012.

[64] V. Ryzhikov, N. Starzhinskiy, L. Gal’Chinetskii, P. Gáshin, D. Kozin, and E. Danshin, "New semiconductor scintillators $\mathrm{ZnSe}(\mathrm{Te}, \mathrm{O})$ and integrated radiation detectors based thereon," IEEE Transactions on Nuclear Science, vol. 48, no. 3, pp. 356-359, 2001.

[65] H. Okada, T. Kawanaka, and S. Ohmoto, "Study on the ZnSe phase diagram by differential thermal analysis," Journal of Crystal Growth, vol. 165, no. 1-2, pp. 31-36, 1996.

[66] M. P. Kulakov, V. D. Kulakovskii, I. B. Savchenko, and A. V. Fadeev, "Phase transition in zinc selenide crystals," Soviet Physics-Solid State, vol. 18, pp. 526-527, 1976.

[67] P. Benetti, I. Dafinei, F. Ferroni et al., "Enriched isotopes for double beta decays experiments: Se82 and Lucifer," in Proceedings of the 12th International Workshop on Separation Phenomena in Liquids and Gases (SPLG '12), pp. 434-837, Paris, France, June 2012.

[68] P. Benetti, I. Dafinei, F. Ferroni et al., "Synthesis of 82Se enriched ZnSe for double beta decay experiments," in Proceedings of the 12th International Workshop on Separation Phenomena in Liquids and Gases (SPLG '12), pp. 438-843, Paris, France, June 2012.

[69] A. Borio di Tigliole, A. Cammi, M. Clemenza, V. Memoli, L. Pattavina, and E. Previtali, "Benchmark evaluation of reactor critical parameters and neutron fluxes distributions at zero power for the TRIGA Mark II reactor of the University of Pavia using the Monte Carlo code MCNP," Progress in Nuclear Energy, vol. 52, no. 5, pp. 494-502, 2010. 
[70] F. Alessandria, R. Ardito, D. R. Artusa et al., "Sensitivity and discovery potential of CUORE to neutrinoless double-beta decay," http://arxiv.org/abs/1109.0494.

[71] D. M. Chernyak, F. A. Danevich, A. Giuliani, E. Olivieri, M. Tenconi, and V. I. Tretyak, "Random coincidence of $2 \nu 2 \beta$ decay events as a background source in bolometric $0 \nu 2 \beta$ decay experiments," The European Physical Journal C, vol. 72, p. 1989, 2012.

[72] J. Menéndez, A. Poves, E. Caurier, and F. Nowacki, "Disassembling the nuclear matrix elements of the neutrinoless $\beta \beta$ decay," Nuclear Physics A, vol. 818, no. 3-4, pp. 139-151, 2009.

[73] A. Faessler, V. Rodin, and F. Simkovic, "Nuclear matrix elements for neutrinoless double-beta decay and double-electron capture," Journal of Physics G, vol. 39, Article ID 124006, 2012.

[74] D.-L. Fang, A. Faessler, V. Rodin, and F. Šimkovic, "Neutrinoless double- $\beta$ decay of deformed nuclei within quasiparticle random-phase approximation with a realistic interaction," Physical Review C, vol. 83, no. 3, Article ID 034320, 2011.

[75] J. Suhonen and O. Civitarese, "Review of the properties of the $0 \nu \beta^{-} \beta^{-}$nuclear matrix elements," Journal of Physics G, vol. 39, Article ID 124005, 2012.

[76] J. Barea, J. Kotilia, and F. Iachello, "Nuclear matrix elements for double-B decay," Physical Review C, vol. 87, Article ID 014315, 2013.

[77] P. K. Rath, R. Chandra, K. Chaturvedi, P. K. Raina, and J. G. Hirsch, "Uncertainties in nuclear transition matrix elements for neutrinoless $\beta \beta$ decay within the projected-Hartree-FockBogoliubov model," Physical Review C, vol. 82, no. 6, Article ID 064310, 2010.

[78] T. R. Rodriguez and G. Martinez-Pinedo, "Energy density functional study of nuclear matrix elements for neutrinoless $\beta \beta$ decay," Physical Review Letters, vol. 105, Article ID 252503, 2010.

[79] J. Kotila and F. Iachello, "Phase-space factors for double- $\beta$ decay," Physical Review C, vol. 85, no. 3, Article ID 034316, 2012. 

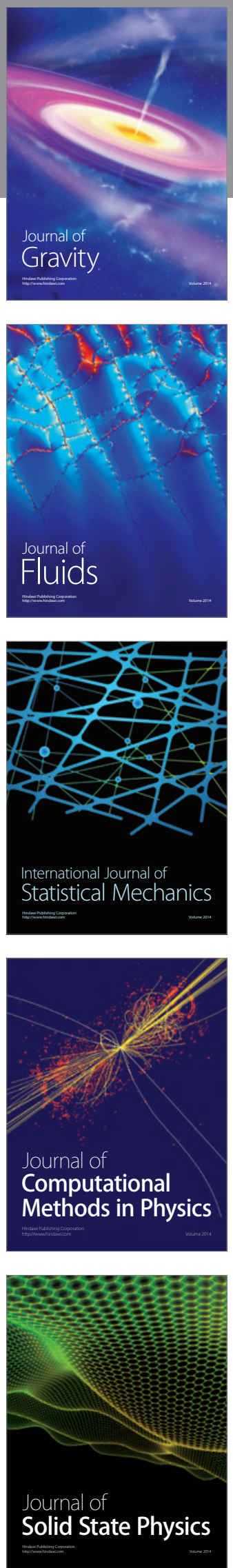

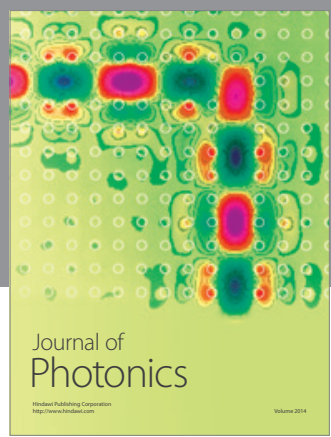

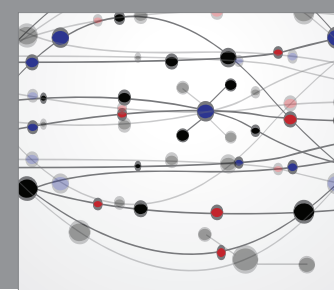

The Scientific World Journal

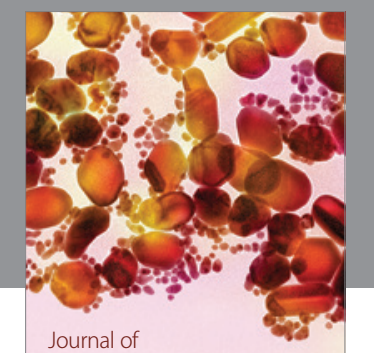

Soft Matter
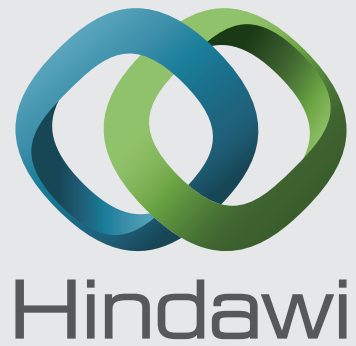

Submit your manuscripts at

http://www.hindawi.com
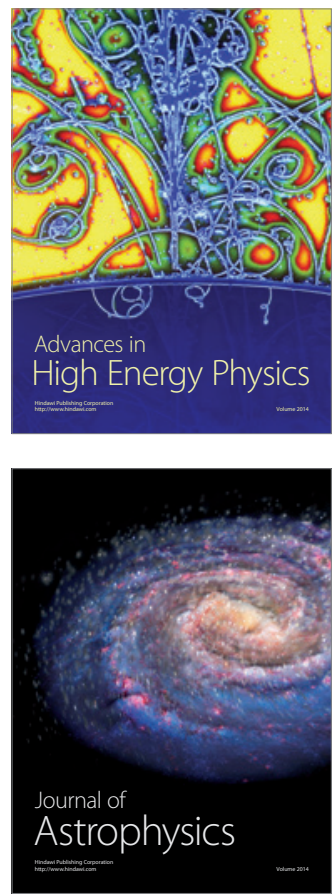
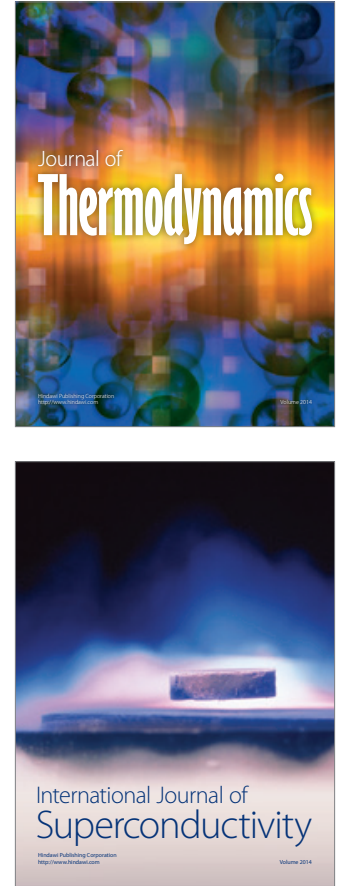
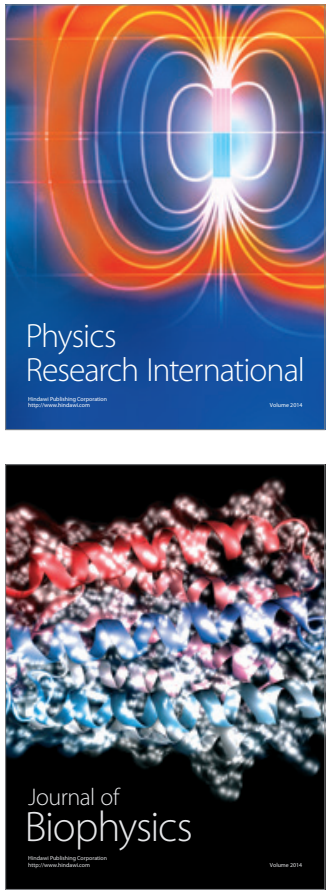
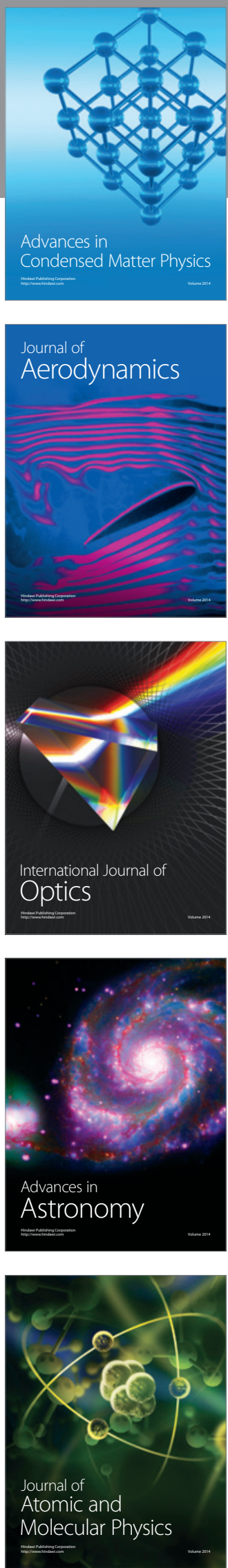